\title{
Effect of Errors in Linear Scaling Relations and Brønsted-Evans-Polanyi Relations on Activity and Selectivity Maps
}

\author{
Jonathan E. Sutton and Dionisios G. Vlachos* \\ Catalysis Center for Energy Innovation and Center for Catalytic Science and Technology, Department \\ of Chemical and Biomolecular Engineering, University of Delaware, Newark, DE 19716
}

\begin{abstract}
We introduce for the first time uncertainty quantification and global sensitivity analysis to assess the effect of error in linear scaling relations (LSRs) and Brønsted-Evans-Polanyi (BEP) relations on activity and selectivity maps of microkinetic models, which have recently been used for in silico prediction of new materials. The method is applied to ethanol hydrodeoxygenation (HDO). Selectivity trends are driven by adsorbate thermochemistry rather than kinetics. Uncertainty quantification calculations show that the most likely location of the maximum conversion can be estimated to within about $10 \mathrm{kcal} / \mathrm{mol}$ in the $\mathrm{C}$ and $\mathrm{O}$ binding energies. Broad selectivity trends are even more robust. Model rates show uncertainties of 2-3 orders of magnitude about the median. Uncertainty in the activity predictions is dominated by that of the LSRs. Our calculations demonstrate that there is a common initial mechanism of ethanol HDO and decomposition, and the stability of decomposition products is crucial to determining the selectivity.
\end{abstract}

Keywords: Ethanol, hydrodeoxygenation, density functional theory, microkinetic modeling, uncertainty quantification, sensitivity analysis, Linear Scaling Relations, Brønsted-Evans-Polanyi correlations

*Corresponding author: e-mail: vlachos@udel.edu; tel. 302-831-2830. 


\section{Introduction}

Model-aided catalyst prediction has been a major topic of research over the last two decades[1,2] due to the promise of developing improved and/or lower cost catalysts and of eliminating intensive empirical testing. To this end, high throughput microkinetic models have been developed for prototypical processes such as ammonia synthesis[3] and decomposition[4], methanation[5, 6], CO hydrogenation[7], methane steam reforming[8], oxygen reduction[9], ethanol decomposition[10], ethylene glycol decomposition[11], and methanol and higher alcohol synthesis.[12]

In the quest of searching for new materials, upon constructing a mechanism, kinetic parameters are estimated without resorting to expensive parameter estimation methods (e.g., density functional theory (DFT)). In practice, atomic binding energies (descriptors) are mapped to species energies via linear scaling relations [13-17] (LSRs) or bond order conservation (BOC) $[18,19]$ and species energies (or heats of reactions, which are their linear combinations, to be more precise) are mapped to reaction activation energies via Brønsted-Evans-Polanyi relations[5, 10, 11, 20-24] (BEPs). These correlations have recently been referred to as first principles-based semi-empirical methods (FPSEM) or first-principles-based screening methods,[25] and due to their simple linear functional form, they are capable of estimating an entire set of kinetic parameters in real time. We term microkinetic (MKM) models using this approach as descriptor-based (in contrast to first-principles-based MKM). The combination of LSRs and BEPs enables one to estimate thermochemistry and activation energies of large reaction networks or for arbitrary materials. The former is essential for modeling complex reaction networks and the latter enables construction of activity and selectivity maps. We provide an example below. These maps in turn elucidate the optimal material properties that could lead to discovery of new catalysts.

These correlations are generally regressed from DFT data, and the regression process invariably introduces additional error above and beyond what is inherently present in the underlying DFT energies (this topic has recently been addressed by Nørskov and coworkers[26] as well as by us[27]). We have previously quantified this error and investigated how it propagates when various FPSEM correlations are combined, [17] and Maestri and Reuter have previously investigated the accuracy of BOC estimates.[19] Further analysis of BEP accuracy has been carried out by Sautet and coworkers.[28-30] While Ulissi et al. have considered the impact of uncertainty in BOC on predictions of ammonia decomposition catalysts with maximum activity,[4] to our knowledge, no prior work has been done on evaluating the impact of errors in LSR and BEP correlations on MKM uncertainty. Total model uncertainty (once ascertained) could be reduced by identifying the most sensitive parameters via a sensitivity analysis and by refining them with improved estimates, in a procedure known as hierarchical refinement.[25] We are unaware of any studies on identifying the most influential parameters in the presence of parametric uncertainty.

In this work, we address these two topics. We first outline the process of developing a high throughput MKM and draw preliminary conclusions into the important parameters and the mechanism. We do this with a model of ethanol hydrodeoxygenation (HDO) as a surrogate that might be used to upgrade biomass into value-added fuels and chemicals. Then we employ uncertainty quantification (UQ) methods to assess the overall contributions of the LSRs and BEPs individually and in combination to the total uncertainty in (1) the 'location' of the maximum conversion and selectivity, i.e., the atomic binding energies and (2) in conversion and selectivity as a function of atomic binding energies. This UQ is carried out with respect to the original model predictions, rather than with respect to experiments. Finally, we employ stochastic reaction path analysis,[27] uncertainty quantification and variance-based global sensitivity analysis (GSA), which explicitly incorporates parametric uncertainty, to explore the mechanism of ethanol HDO at representative catalyst formulations.

\section{Methods}

\section{Density Functional Theory Calculations}

Select DFT calculations were carried out in order to parameterize BEP relations for reactions in the water-gas mechanism and for the oxidative dehydrogenation of terminal $\mathrm{OH}$ groups by coadsorbed $\mathrm{O}^{*}$ or 
$\mathrm{OH}^{*}$ on the close-packed facets of $\mathrm{Co}, \mathrm{Ni}, \mathrm{Pd}, \mathrm{Pt}, \mathrm{Rh}$, and $\mathrm{Ru}$. The results of the DFT calculations and the BEP correlations are given in the Supporting Information (SI). We have chosen to employ the closepacked facets rather than other, more open facets (e.g., steps or open terraces) that are often the active phase to reduce the number of DFT calculations. This simplification should not alter our general conclusions because LSR and BEP correlations for terraces and steps are typically similar, with slopes and intercepts that differ by a small amount.[13,31] Furthermore, by scanning over a wide range of atomic binding energies, we implicitly include the more open facets, although with some systematic error. Overall, we believe that close-packed facets provide a reasonable starting point for a qualitative identification of promising materials. More quantitative predictions must be made with higher fidelity models, such as kinetic Monte Carlo simulations, of various facets. One can view this an implementation of the principle of hierarchical model refinement.[25, 32]

DFT calculations were performed with the SIESTA code.[33] Computational parameters followed a method employed in previous work.[34] We used 3x3 unit cells with four metal layers (bottom two layers frozen) and $15 \AA$ vacuum slabs with a 3x3x1 Monkhorst-Pack k-point mesh and the PBE functional.[35] This functional tends to result in higher binding energies compared to the best available experimental data.[36] The systematic errors in this functional (note that systematic errors are common to all the GGAtype functionals) will result in systematic errors in model predictions with respect to experiment. Geometries were considered relaxed when a force tolerance of $0.1 \mathrm{eV} / \AA$ was reached. This force tolerance was chosen to reduce the computational burden associated with the large number of calculations required for training the semi-empirical correlations. Our structures obtained with this tolerance closely match the structures obtained with a more rigorous force tolerance $(0.05 \mathrm{eV} / \AA)$ and finer k-point mesh $(5 \times 5 \times 1$ for the same supercell).[15] We expect that this similarity extends to the energies as well and errors in the energies arising from the less rigorous force tolerance will be smaller than the errors arising from the correlations fit to the training data. Transition states were located via a constrained optimization technique[37] coupled with an automatic transition state search algorithm.[38] Prior work has shown that this procedure yields reasonable estimates of the transition state geometries that are sufficient for parameterizing the BEP relations required for this work.

\section{Microkinetic Modeling (MKM)}

The high throughput MKM of ethanol HDO in this work was based on our prior model of ethanol steam reforming on Pt.[25, 39] To our earlier model we added oxidative dehydrogenation reactions for the removal of $\mathrm{H}$ by coadsorbed $\mathrm{O}^{*}$ or $\mathrm{OH}^{*}$, i.e., $\mathrm{RH}^{*}+\mathrm{O}^{*}=\mathrm{R}^{*}+\mathrm{OH}^{*}$ and $\mathrm{RH}^{*}+\mathrm{OH}^{*}=\mathrm{R}^{*}+\mathrm{OH}_{2} *$, where $\mathrm{R}$ is any intermediate of ethanol in proximity with the surface to enable $\mathrm{H}$ transfer. In total, our revised mechanism has 67 species and 300 reactions.

To rapidly parameterize the microkinetic model, two types of semi-empirical correlations were employed: extended LSRs (ELSRs) for species energies and BEP relations for activation energies. These correlations are obtained by performing DFT calculations for a wide variety of intermediates and transition states and then fitting correlations to the training data. Correlation errors are estimated from the deviations between correlation estimates and the corresponding DFT data.

Species energies were estimated from carbon and oxygen atomic binding energies via ELSRs, with parameters taken from our prior work.[17] The ELSRs have the following functional form (adapted from Eq. 25, Ref. [17]):

$$
\hat{E}_{M_{2}}=\hat{E}_{M_{1}}+\sum_{j} \sum_{i} \gamma_{j}\left(E_{i, M_{2}}-E_{i, M_{1}}\right)
$$

where $\hat{E}_{M_{2}}$ is the estimate of the binding energy on metal 2, $\hat{E}_{M_{1}}$ is the reference binding energy on metal $1, \gamma_{j}$ is the slope (obtained from regressing DFT data) approximately measuring the contribution of the metal to the electron density around heteroatom $j, E_{i, M_{2}}$ is the atomic binding energy of the type $i$ heteroatom on metal 2, and $E_{i, M_{1}}$ is its equivalent for metal 1 . The heteroatom index $j$ is for convenience, as the same heteroatom type may be repeated (e.g., two distinct $\mathrm{C}$ binding centers). The reference 
energies for the ELSRs were taken from our prior DFT-based temperature-corrected thermochemistry on $\mathrm{Pt},[25,39]$ which in turn were based on DFT calculations by Salciccioli et al.[15] Adsorbate lateral interactions were adjusted as atomic binding energies vary using the method of Salciccioli and Vlachos.[11]

Activation energies were estimated using BEP relations for activation of ethanol on a variety of transition metals. These BEP relations are a combination of prior work[34] and new calculations (see the SI). Consistent with our findings on Pt[39], oxidative dehydrogenation reactions were assumed to follow the corresponding thermal dehydrogenation homologous series, with the sole exception of the dehydrogenation of terminal $\mathrm{OH}$ groups by adsorbed $\mathrm{OH}^{*}$ (in these reactions hydrogen bonding plays a key role and barriers are very small or absent). Owing to the importance of the water-gas shift mechanism in removing adsorbed $\mathrm{O}^{*}$ and $\mathrm{OH}^{*}$ and oxidizing $\mathrm{CO}^{*}$, we also developed BEP relations specifically for the water-gas shift chemistry (see the SI). For convenience, we summarize the LSR parameters in Table 1 and the BEP parameters in Table 2.

Table 1. Extended linear scaling relations (ELSR) slopes (reference binding energies were from Pt). The overall uncertainty in the LSR estimates was estimated as $0.023 \pm 0.308 \mathrm{eV}$ (mean \pm s.d.). The water LSR slope was arbitrarily set to 0.1 (weak dependence on the metal) and was assumed to hold for closed-shell alcohols as well. LSR parameters taken from Sutton and Vlachos.[17]

\begin{tabular}{cc} 
Binding Mode & Slope \\
\hline $\mathrm{CH}_{3}$ & 0.4451 \\
$\mathrm{CH}_{2}$ & 0.5663 \\
$\mathrm{CH}$ & 0.8182 \\
$\mathrm{CH}_{2} \mathrm{OH}$ & 0.4151 \\
$\mathrm{CHOH}$ & 0.7140 \\
$\mathrm{COH}$ & 0.6586 \\
$\mathrm{CH}_{2} \mathrm{O}$ & \\
(via $\mathrm{C}$ ) & 0.2561 \\
$\mathrm{CH} \mathrm{O}_{2} \mathrm{O}$ & \\
(via $\mathrm{O}$ ) & 0.1426 \\
$\mathrm{CHO}$ & 0.6571 \\
$\mathrm{CO}$ & 0.4174 \\
$\mathrm{OH}$ & 0.3672 \\
$\mathrm{H}_{2} \mathrm{O}$ & 0.1000
\end{tabular}

Table 2. BEP parameters and standard deviations in the errors (the means were assumed to be zero).

\begin{tabular}{|c|c|c|c|c|}
\hline Homologous Series & Slope & Intercept $(\mathrm{eV})$ & Std Dev $(\mathrm{eV})$ & Reference \\
\hline C-H cleavage ${ }^{1}$ & 0.5860 & 0.7840 & 0.2882 & {$[34]$} \\
\hline O-H cleavage ${ }^{1}$ & 0.2815 & 0.7550 & 0.1454 & {$[34]$} \\
\hline C-C cleavage ${ }^{1}$ & 0.7106 & 1.3031 & 0.4138 & [34] \\
\hline C-OH cleavage ${ }^{1}$ & 0.5801 & 1.2177 & 0.3916 & [34] \\
\hline C-O cleavage ${ }^{1}$ & 0.8125 & 1.4675 & 0.3945 & [34] \\
\hline $\mathrm{CH}_{3} \mathrm{CH}_{2} \mathrm{OH}, \alpha \mathrm{C}-\mathrm{H}^{2}$ & 0.6954 & 0.9271 & 0.0416 & [34] \\
\hline $\mathrm{CH}_{3} \mathrm{CH}_{2} \mathrm{O}-\mathrm{H}^{2}$ & 0.1872 & 0.8487 & 0.0770 & [34] \\
\hline $\mathrm{CH}_{3} \mathrm{COH}, \mathrm{O}-\mathrm{H}^{2}$ & 0.2352 & 0.7472 & 0.1206 & {$[34]$} \\
\hline $\mathrm{CH}_{3} \mathrm{C}-\mathrm{OH}^{2}$ & 0.5284 & 0.9458 & 0.1012 & [34] \\
\hline $\mathrm{CH}_{3}-\mathrm{CO}^{2}$ & 0.7794 & 1.2493 & 0.1832 & [34] \\
\hline $\mathrm{CH}_{3} \mathrm{C}-\mathrm{O}$ & 1.5016 & 1.6535 & 0.2031 & [34] \\
\hline $\mathrm{CH}_{2} \mathrm{CO}, \beta \mathrm{C}-\mathrm{H}^{2}$ & 1.1049 & 1.0611 & 0.2132 & [34] \\
\hline $\mathrm{CH}_{2}-\mathrm{CO}^{2}$ & 0.7900 & 1.1023 & 0.3225 & [34] \\
\hline
\end{tabular}




$\begin{array}{lllll}\mathrm{CH}-\mathrm{CO}^{2} & 0.5333 & 0.5815 & 0.0730 & {[34]} \\ \mathrm{H}_{2} \mathrm{O}, \mathrm{O}-\mathrm{H}^{2,3} & 0 & 0.8734 & 0.1015 & \text { This work } \\ \mathrm{O}-\mathrm{H}^{2,3} & 0 & 1.2311 & 0.3310 & \text { This work } \\ \mathrm{CO}+\mathrm{O}^{2,4} & 0.2816 & 0.9701 & 0.0911 & \text { This work } \\ \mathrm{CO}+\mathrm{OH}^{2} & 0.7455 & 0.6565 & 0.0505 & \text { This work } \\ \mathrm{COO}-\mathrm{H}^{2,3} & 0 & 0.8942 & 0.1221 & \text { This work } \\ \mathrm{O}-\mathrm{H} \mathrm{ODH} & 0.3430 & 0.7342 & \text { This work } \\ \text { 1. Universal BEP for a variety of reactants on all metals. } & & \\ \text { 2. Reaction-specific BEP as a function of metal. } & & \\ \text { 3. Slope was not statistically significant and close to zero, so the best-fit BEP was assumed to just be the mean activation } \\ \text { 4. Exergy (slope explicitly set to zero). }\end{array}$

The correlations in Table 1 and Table 2 were obtained via ordinary least squares minimization in order to minimize the average deviation from the training data. Generally, the correlation parameters retain their physical meaning. For instance, the ELSR slope is based on the fraction of heteroatom electron density provided by the surface, and the BEP slope is a measure of the earliness or lateness of the transition state (ranging from 0 to 1 ). Very small (0) or very large (1) BEP slopes merely imply that the transition state is identical to the reactant or product, respectively. We have previously found that BEP slopes for the same set of reactions written in opposite directions must sum to unity,[24] implying that whether a transition state is reactant-like or product-like is entirely dependent on the choice of convention for the reactant and product. Occasionally, however, the least-squares-derived parameters exceed the accepted range, potentially for two possible reasons. For small deviations (smaller than about 0.1 for BEP slopes), it is likely a reflection of the known tendency for small molecules to have more significant geometric relaxations than large molecules.[40] For larger deviations (e.g., the slope of 1.5 for $\mathrm{CH}_{3} \mathrm{CO}$ C-O scission), the more likely source is that two or more distinct homologous series have been included in the regression. For this specific case, if the points for the non-oxophilic metals $\mathrm{Pd}$ and $\mathrm{Pt}$ are excluded from the points for the more oxophilic metals $\mathrm{Co}, \mathrm{Ni}, \mathrm{Rh}$, and $\mathrm{Ru}$, the slope decreases to about 1 .

This microkinetic model was then solved using a triangular mesh (to maximize the density of mesh points for a given spacing and to simplify discrete binning of points for later analysis of the distributions of the locations of maximum conversion and selectivity) of carbon and oxygen binding energies to identify trends in activity, selectivity, and adsorbate coverage. Maps were generated with a spacing of 2 $\mathrm{kcal} / \mathrm{mol}$ between mesh points for the nominal model (in the absence of uncertainty) and with a spacing of $3 \mathrm{kcal} / \mathrm{mol}$ for the UQ calculations. The coarser mesh was required to reduce the high computational burden associated with the UQ calculations.

\section{Uncertainty Quantification and Global Sensitivity Analysis}

One of the key aspects of this work is the explicit incorporation of UQ and GSA. UQ assists in assessing the inherent variability of model predictions and GSA provides quantitative insight into which parameters are the principal source of that variability.

UQ calculations were performed by assuming that errors in the LSRs and BEPs arise from the least squares regression and not from the underlying DFT data, as we expect that the linear correlation errors will have more impact on model uncertainty than the DFT errors. The deviations between least squares regression estimates and the corresponding actual values are taken to follow the same normal distribution, and individual errors are independent of each other (this also implies no correlations exist between them). Following these two standard assumptions, we sample the error in each species or reaction energy using the distributions of the deviations between the actual DFT data and the corresponding correlation estimates, i.e., using a normal distribution that describes the 'scatter' of each correlation. The statistics of the distributions are summarized in Tables 1 and 2.

The UQ and GSA methodology maintains thermodynamic consistency and proceeds as follows. We first estimate species energies with the LSRs and then add a perturbation sampled from the uncertainty distribution. This results in perturbed species energies. Reaction energies are calculated from the 
perturbed species energies and automatically incorporate LSR uncertainty. Given that species energies form a thermodynamic basis for reaction networks which consist of more reactions than species, this ensures that all pathways are thermodynamically consistent.[41] We have previously shown that BEP correlation estimates are subject to two types of error: errors related to the reaction energy inputs and errors intrinsic to the regression process.[17] For sampling the BEP error, employing the perturbed reaction energies as input to the BEP relation automatically accounts for the first source of error. The second source of error is then sampled directly, and this perturbation is added to the estimated forward activation energy. Reverse activation energies are calculated from the forward activation energies and the reaction energies. This approach ensures that the kinetics is also consistent with the thermodynamics of the entire network.[41] By applying perturbations at each stage of the parameters' estimation process, the LSR uncertainty propagates to the activation energy as described in our previous work. [17] The combined uncertainty in turn propagates into the solution of the MKM, including coverages and reaction rates. One of our goals with the UQ is specifically to evaluate the overall impact of errors in each parameter type on the distribution of model responses.

Error distributions for the LSRs have previously been estimated[17] and are used without further adjustment in this work. BEP error distributions for the thermal decomposition reactions were also estimated in prior work[34], and error distributions for the remaining homologous series are estimated in the SI. In performing UQ calculations, the estimated parameters are perturbed within their uncertainty range, and the model is solved. After a sufficient number of replicates (many thousands at least), it is possible to construct a distribution for each of the model responses (rates, selectivities, etc.). We characterize these distributions with representative percentiles: the median and $\pm 34 \%$ bounds (for a normal distribution these correspond to the mean bracketed by one standard deviation).

We also carry out GSA calculations using a variance-based method.[42, 43] We sample points using the radial (or iterated one-factor-at-a-time) method[44, 45], which involves drawing two independent perturbation samples (called the $\mathbf{A}$ and $\mathbf{B}$ vectors). The algorithm then involves solving the model at each of the two independent samples as well as at perturbation samples constructed by replacing one perturbed value at a time in the first set with the corresponding value from the second set (termed the $\mathbf{A}_{\mathbf{B}}$ vector). The fraction of the total model variance attributable to a given parameter can then be estimated using an ANOVA decomposition on the model output (additional details are given in the SI; GSA methods for kinetic modeling have been thoroughly reviewed by Saltelli et al.[46]). We outline the mathematical foundations in the SI.

To efficiently obtain well-converged sensitivity indices at a reduced cost, we employ a two-step procedure. First, we conduct a screening calculation wherein we perturb all parameters but employ fewer total repetitions. From this screening run, parameters with a total sensitivity index larger than 0.02 (i.e., this parameter in combination with all others contributes to at least $2 \%$ of the total variance) are selected for further convergence; all others are subsequently fixed at their nominal values. The second step is to repeat the GSA calculation for the influential parameters using a larger number of repetitions. We have compared the second set to the first and verified qualitative agreement (details in SI).

\section{Activity, Selectivity, and Coverage Maps for the Nominal Model}

Before investigating the effect of uncertainty on model responses, we first demonstrate the predictions made by high throughput MKM. In particular, we construct activity, selectivity, and coverage maps to identify trends in model responses as a function of carbon and oxygen atomic binding energies. Reactor conditions were chosen to ensure relatively low, but not necessarily differential conversions. 

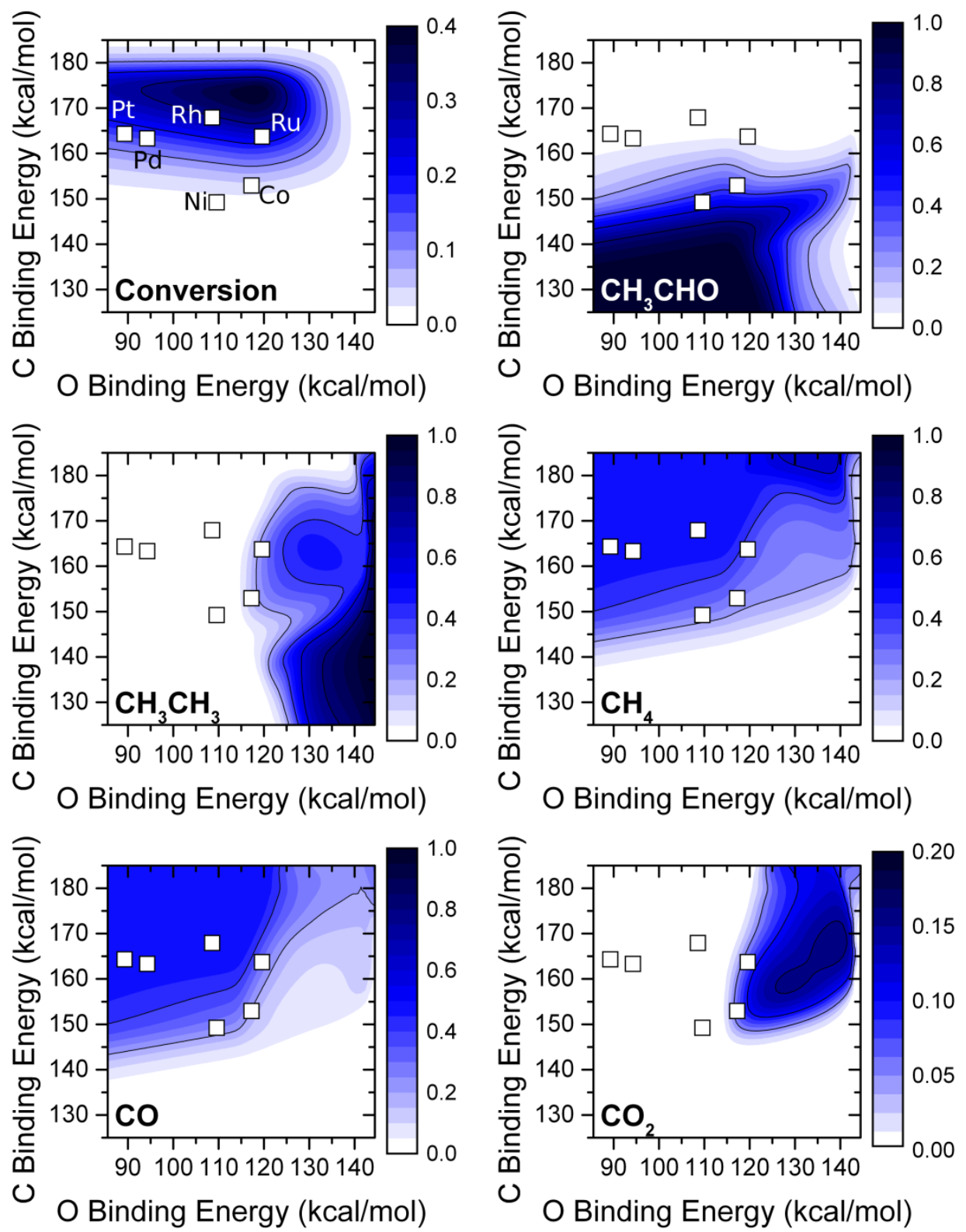

Figure 1. Nominal activity and carbon selectivity maps for ethanol HDO as a function of carbon (C) and oxygen (O) binding energy. Reaction conditions are $573 \mathrm{~K}, 1 \mathrm{~atm}, 12.5 \mathrm{~mol} \%$ ethanol, $37.5 \mathrm{~mol} \% \mathrm{H}_{2}$, bal. $\mathrm{He}$ with $\mathrm{S} / \mathrm{V}=10 \mathrm{~cm}^{-1}$, flow.rate of 1.77 standard $\mathrm{cm}^{3} / \mathrm{min}$, and volume of $1 \mathrm{~cm}^{3}$. Values are fractional. Atomic binding energies for the six closepacked metals are superimposed as white squares on the maps.

Figure 1 shows that activity is maximized at moderate to high $\mathrm{C}$ binding energies and moderate $\mathrm{O}$ binding energies, with the $\mathrm{C}$ binding energy being more important. Four of the single metals lie in the region of moderate to high activity, with $\mathrm{Rh}$ and $\mathrm{Ru}$ being closest to the maximum conversion. With the exception of $\mathrm{O}$ binding energies higher than $140 \mathrm{kcal} / \mathrm{mol}$, there are enough vacant sites present (at least 20\%; Figure 2), and the binding energy, rather than site availability controls the activity. At the high $\mathrm{O}$ binding energies (very oxophilic catalysts), the rapid build-up of $\mathrm{O}$ blocks sites, and site availability becomes a limiting factor. Activity is low in the acetaldehyde region because ethanol binds too weakly and desorbs fast, but as the $\mathrm{C}$ binding energy increases, so does the activity. The impact of the $\mathrm{C}$ binding energy relative to the $\mathrm{O}$ binding energy is consistent with previous findings that the mean binding energy of ethanol-derived species with respect to gas-phase ethanol generally increases with $\mathrm{C}$ binding energy, 
whereas the $\mathrm{O}$ binding energy has a secondary effect.[34] Dehydrogenation to acetaldehyde is preferred at low $\mathrm{O}$ and $\mathrm{C}$ binding energies. Ethane selectivity is maximized at low $\mathrm{C}$ and high $\mathrm{O}$ binding energies, but it is also prevalent at moderate $\mathrm{C}$ and high $\mathrm{O}$ binding energies. As the $\mathrm{C}$ binding energy increases, the product distribution switches from acetaldehyde and ethane to methane and $\mathrm{CO}$ (low $\mathrm{O}$ binding energies) and to a mixture of ethane, methane, and $\mathrm{CO}_{2}$ (high $\mathrm{O}$ binding energies). At high $\mathrm{O}$ and $\mathrm{C}$ binding energies, both $\mathrm{C}-\mathrm{C}$ and $\mathrm{C}-\mathrm{O}$ cracking are active; most of the $\mathrm{CO}$ is directly oxidized to $\mathrm{CO}_{2}$ with $\mathrm{O}$ * produced from the $\mathrm{C}-\mathrm{O}$ cracking. At very high $\mathrm{C}$ binding energies, methane is the dominant hydrocarbon. In the region of high activity, catalytic cracking of ethanol, $\mathrm{CH}_{3} \mathrm{CH}_{2} \mathrm{OH} \rightarrow \mathrm{CH}_{4}+\mathrm{CO}+\mathrm{H}_{2}$, dominates. Ethane, an HDO product, can be produced with high selectivity but at very low conversion. The HDO region is limited to fairly oxophilic materials, in qualitative agreement with prior ethylene glycol predictions.[11] Under no set of atomic binding energies do we see appreciable $\mathrm{C}$ buildup as the concentration of $\mathrm{H}_{2}$ in the feed is sufficient to ensure complete removal of C-containing fragments.

The selectivity trends in Figure 1 are driven primarily by adsorbate thermochemistry (i.e., atomic and species binding energies), rather than kinetics. $\mathrm{C}-\mathrm{C}$ cracking is at the top left (low $\mathrm{O}$ and high $\mathrm{C}$ binding energies). Consistent with the LSR principle, small species binding through $\mathrm{C}$ (e.g., $\mathrm{CO}^{*}$ and $\mathrm{CH}_{\mathrm{x}}{ }^{*}$, see Figure 2 for surface coverages) are preferred in this region where $\mathrm{C}$ binding energies are high. Similarly, dehydrogenation is at the bottom left (low $\mathrm{C}$ and $\mathrm{O}$ binding energies) where acetaldehyde binds too weakly to undergo further reaction once formed. The principal surface species in this region is $\mathrm{H}^{*}$, formed both from the dehydrogenation of ethanol and the presence of $\mathrm{H}_{2}$ in the feed. Finally, C-O cracking to ethane and water is on the lower right (high $\mathrm{O}$ binding energies) where O-containing species are more stable than $\mathrm{C}$-containing species (the $\mathrm{C}_{2}$ hydrocarbons formed rapidly hydrogenate to ethane and desorb). As the $\mathrm{C}$ binding energy increases, $\mathrm{C}-\mathrm{C}$ cracking also becomes important, and $\mathrm{O} *$ is removed via $\mathrm{CO}^{*}$ oxidation (note the high coverages of both $\mathrm{CO}^{*}$ and $\mathrm{O}^{*}$ ). At the extreme top right, when the $\mathrm{C}$ binding energy is high enough to bind the $\mathrm{C}_{2}$ fragments, further decomposition via C-C cracking is possible, and methane, rather than ethane is formed (here the $\mathrm{H}_{2}$ co-feed is vital in the methane formation process). Importantly, single metals all lie in the region where $\mathrm{C}-\mathrm{C}$ cracking is preferred, but $\mathrm{Ru}$ and Co exhibit some deoxygenation activity (C-O cracking). $\mathrm{Ru}$ in particular is an interesting material as it is known to be a good HDO catalyst, e.g.[47]. 

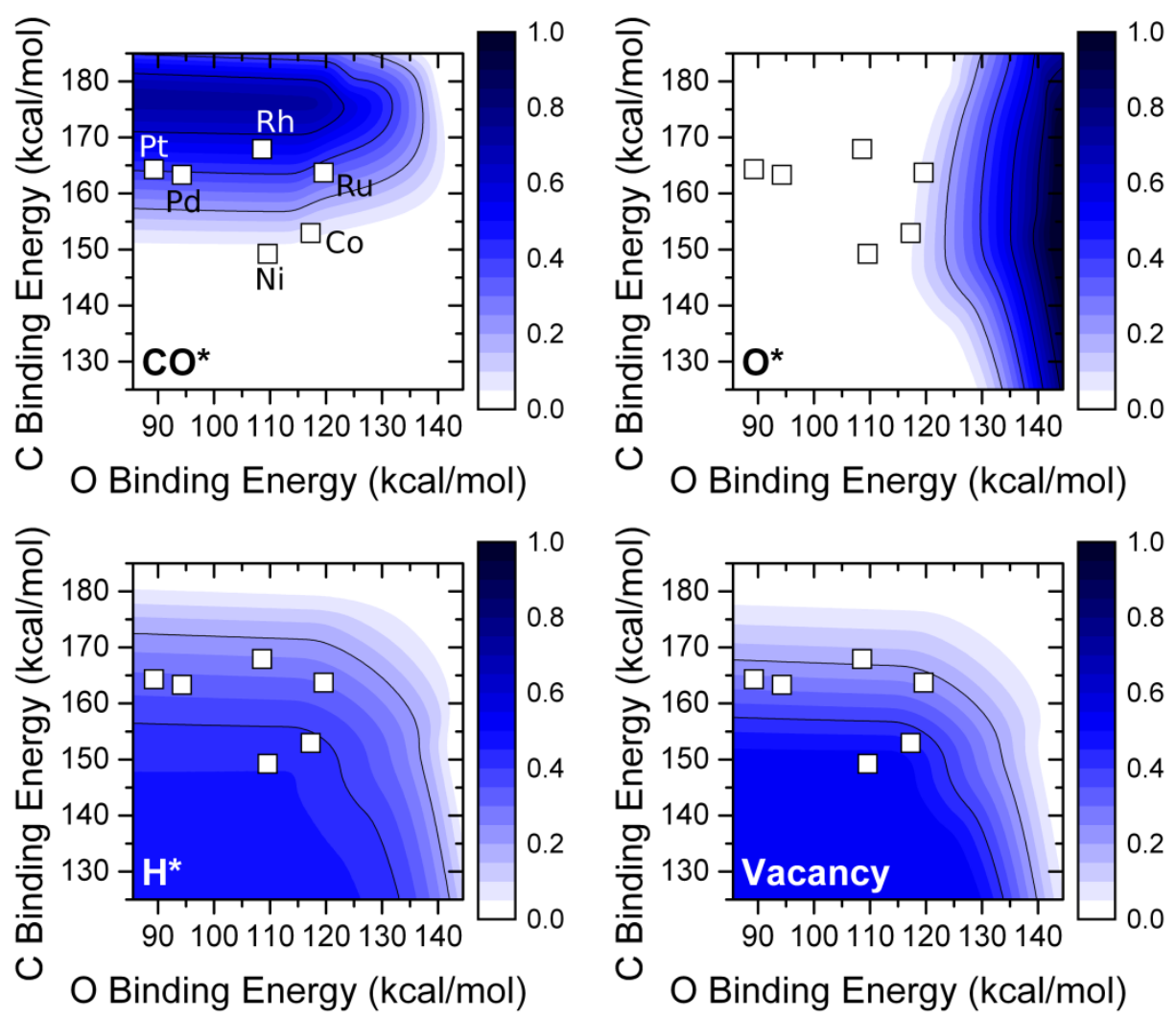

Figure 2. Coverages for dominant species of nominal model. Reaction conditions are the same as in Figure 1 . Very high $\mathrm{C}$ binding energies $(\sim 185 \mathrm{kcal} / \mathrm{mol})$ also have significant quantities of $\mathrm{CCO}^{*}$ (low to moderate $\mathrm{O}$ binding energies) and $\mathrm{CH}^{*}$ (high $\mathrm{O}$ binding energies).

\section{Contributions of LSR and BEP Errors to Uncertainty}

A key question is the reliability of the model predictions. In particular, we wish to investigate the impact of the errors from the LSRs and BEPs on the locations of the maximum activity and selectivity and on the uncertainties in the rates and selectivities. The first is important for guiding experimental tests of proposed catalyst formulations as it gives an estimated range for the atomic binding energies that might give the best performance. The second is vital for understanding whether model rates and selectivities are consistent with experimental data. 

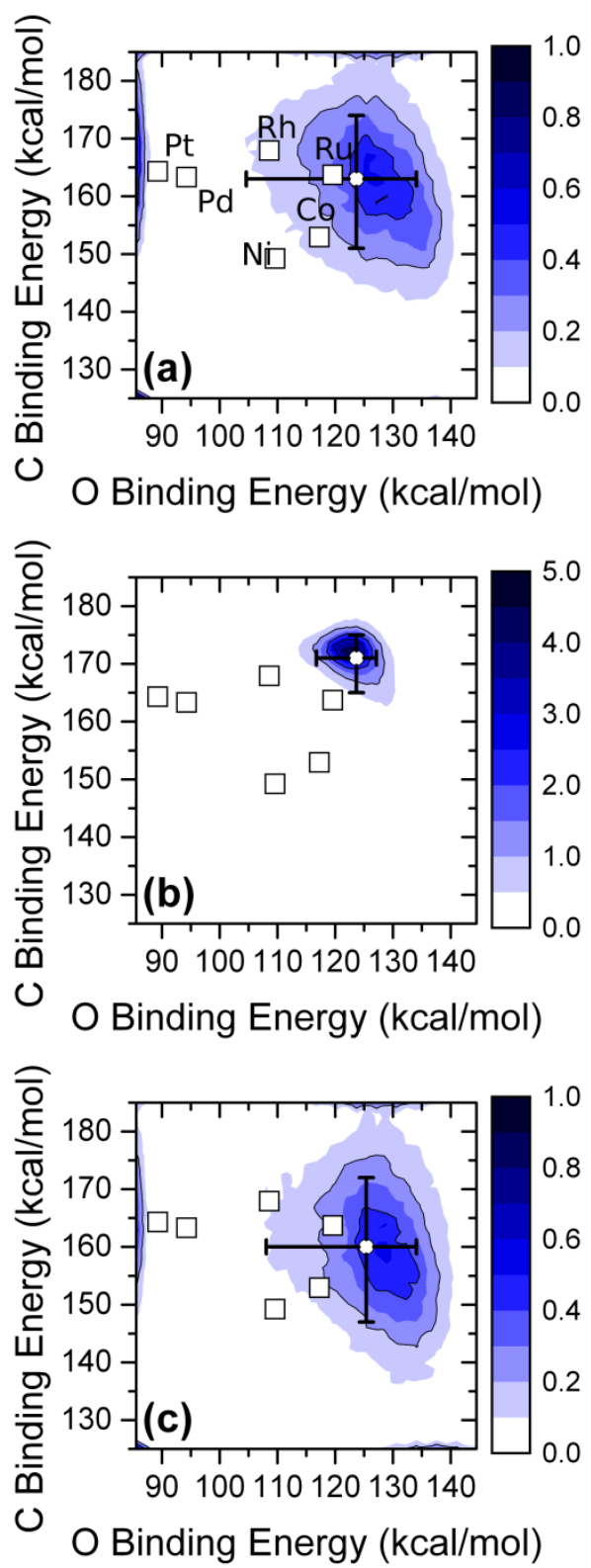

Figure 3. Relative (percent) frequencies with which individual binding energies are the location of the maximum conversion for uncertainties in (a) the linear scaling relations, (b) the Brønsted-Evans-Polanyi relations, and (c) the combination of the two. Reaction conditions are the same as in Figure 1. The median $\mathrm{C}$ and $\mathrm{O}$ binding energies where the conversion is maximized are shown as small open white circles. Error bars on the white circles represent $\pm 34 \%$ confidence intervals in each direction.

We begin by investigating the distribution of the location of maximum conversion, following the procedure of Ulissi et al.[4] Figure 3 shows that uncertainty in the LSRs dominates the distribution of the location of maximum conversion. The distribution of the location of maximum conversion is broad when only the LSR uncertainty is included (Figure 3a). When only BEP uncertainty is employed, the location of maximum conversion is highly localized (Figure $3 \mathrm{~b}$ ) and close to the maximum conversion in the nominal model (Figure 1). When both are combined (Figure 3c), the distribution is very similar to the LSR-only distribution but also shifted somewhat (compare the medians of the binding energies with the maximum conversion indicated by the black squares), indicating that there is a slight nonlinear interaction between the two uncertainties. 
The most likely location for the maximum conversion is significantly shifted from the nominal maximum when ELSR uncertainties are present due to systematic errors in binding energies estimated using the ELSRs.[17]The finding that ELSRs contribute more to the model uncertainty than does the BEP uncertainty was unexpected, owing to the relative sizes of the ELSR and BEP errors. BEP errors can be larger than $1 \mathrm{eV}$, whereas ELSR errors are around an order of magnitude smaller. Furthermore, the BEP error dominates the combined ELSR and BEP uncertainty when the two correlations are used together.[17] We believe that the large contribution of the ELSRs to the model uncertainty is due primarily to the greater importance of adsorbate thermochemistry relative to the kinetics, even in the unperturbed model. The barriers are low enough at the current conditions that other species-related effects are more important. For example, in our previous model of ethanol steam reforming, the rate determining step was found to be the initial dehydrogenation of ethanol because the concentration of adsorbed ethanol was very low.[39] Other compensating effects, such as a large number of parallel reactions or adsorbate interactions (our adsorbate interactions are a function of the atomic binding energies), could also contribute to rendering activation barriers low enough that they do not inhibit overall model rates to the same degree that species binding energies do.

In the case of the combined error distribution, the uncertainty in the location of the binding energy with the highest activity is around $10 \mathrm{kcal} / \mathrm{mol}$, or about $5-10 \%$ of the value of the binding energy (note that the uncertainty is asymmetric, due to the underlying asymmetry of the distribution). The robustness in predicting materials properties for maximum activity is consistent with our earlier work[4] on ammonia decomposition; there, the work focused primarily on uncertainty in pre-exponentials due to using bondorder-conservation for parametrization, which, unlike this work, lacks well-quantifiable errors in energetics.

The locations of maximum selectivity to various major products are extremely robust. Maximum selectivities are achieved at the corners of the map (see Table 3 for median binding energies where selectivity is maximized). For instance, dehydrogenation is maximized at low $\mathrm{C}$ and $\mathrm{O}$ binding energies, $\mathrm{C}-\mathrm{C}$ cracking is maximized at high $\mathrm{C}$ binding energies, and $\mathrm{C}-\mathrm{O}$ cracking is maximized at high $\mathrm{O}$ binding energies. To the best of our knowledge, this is the first time that the effect of errors of fast-screening parameter-estimation methods on selectivity is addressed. Overall, the errors in the correlations have little impact on which materials are predicted to have the highest selectivity, which is an important outcome of this work.

Table 3. Median carbon (C) and oxygen (O) binding energies (kcal/mol) of the location of maximum selectivity to major products for each of the sources of error.

\begin{tabular}{cllllll} 
& LSR & \multicolumn{3}{c}{ BEP } & LSR+BEP \\
Species & $\mathrm{C}$ & $\mathrm{O}$ & $\mathrm{C}$ & $\mathrm{O}$ & $\mathrm{C}$ & $\mathrm{O}$ \\
\hline $\mathrm{CH}_{3} \mathrm{CHO}$ & 125.00 & 85.60 & 125.00 & 85.60 & 125.00 & 89.02 \\
$\mathrm{CH}_{3} \mathrm{CH}_{3}$ & 139.00 & 144.45 & 133.00 & 144.45 & 135.00 & 144.45 \\
$\mathrm{CH}_{4}$ & 183.00 & 127.12 & 179.00 & 128.86 & 178.00 & 123.66 \\
$\mathrm{CO}$ & 185.00 & 85.60 & 185.00 & 85.60 & 184.00 & 85.56 \\
$\mathrm{CO}_{2}$ & 174.00 & 134.05 & 168.00 & 130.59 & 171.00 & 134.05
\end{tabular}




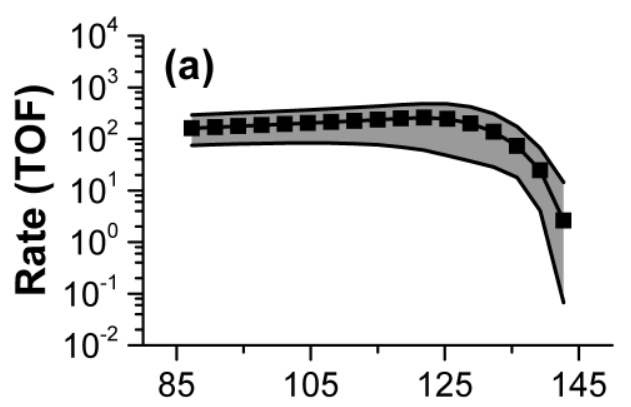

Oxygen Binding Energy (kcal/mol)

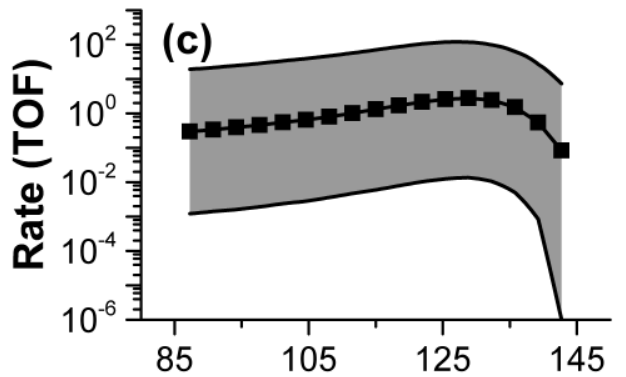

Oxygen Binding Energy (kcal/mol)

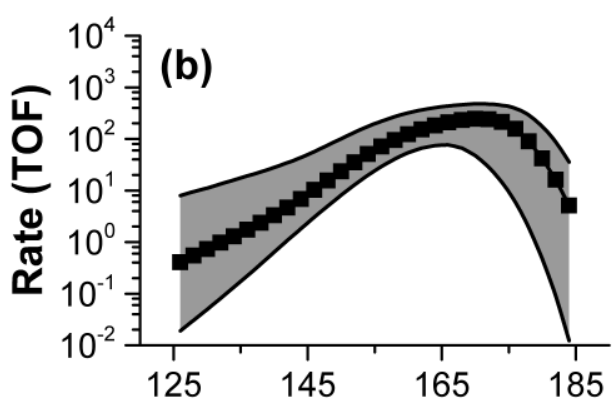

Carbon Binding Energy (kcal/mol)

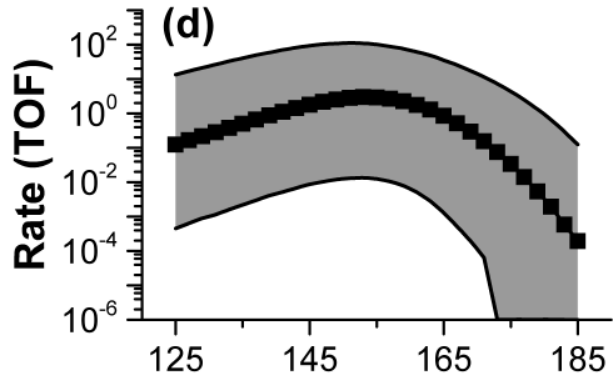

Carbon Binding Energy (kcal/mol)

Figure 4. Uncertainty distributions for reaction rate (turnover frequency in $\mathrm{s}^{-1}$ ) as a function of oxygen binding energy at a carbon binding energy of $150 \mathrm{kcal} / \mathrm{mol}(\mathrm{a}, \mathrm{c})$ and as a function of carbon binding energy at an oxygen binding energy of $130 \mathrm{kcal} / \mathrm{mol}$ (b, d). Panels (a) and (b) are for BEP uncertainty, and panels (c) and (d) are for combined LSR and BEP uncertainty (LSR-only uncertainty plots are given in the SI due to their similarity to the combined distribution plots). Black lines with symbols are the median $\left(50^{\text {th }}\right.$ percentile) responses, and solid black lines delineate the shaded $\pm 34 \%$ envelope (i.e., $68 \%$ of all responses are within these bounds). For a normal distribution, these percentiles are the mean \pm 1 s.d. After post-processing, some rates were calculated to be identically zero. These were set to $10^{-6}$, which is an order of magnitude smaller than all other lower bounds (e.g., panels $\mathrm{c}$ and $\mathrm{d}$ at the high binding energies).

In addition to the location of the maximum conversion, we also explore the uncertainty distributions for activity and selectivity. Figure 4 examines the uncertainty distributions for the rate (black lines and symbols). The BEP-driven uncertainty is typically less than one order of magnitude around the median and larger near the extreme binding energies (Figure 4a and b). The LSR-driven uncertainty is around 2 and occasionally 3 orders of magnitude around the median (Figure S1), and the LSR uncertainty dominates the combined uncertainty (Figure $4 \mathrm{c}$ and $\mathrm{d}$ ). The uncertainty envelope in carbon selectivities for major products (Figure 5) is generally fairly large. 


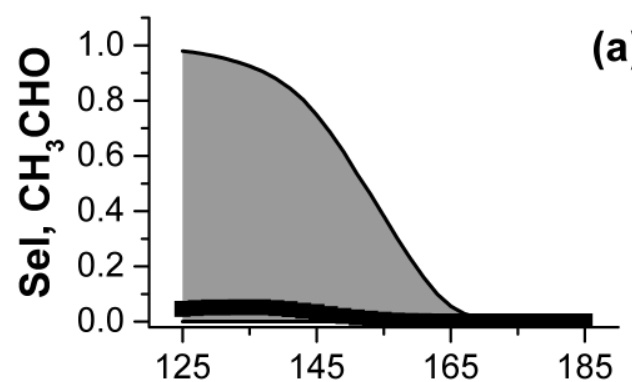

Carbon Binding Energy (kcal/mol)

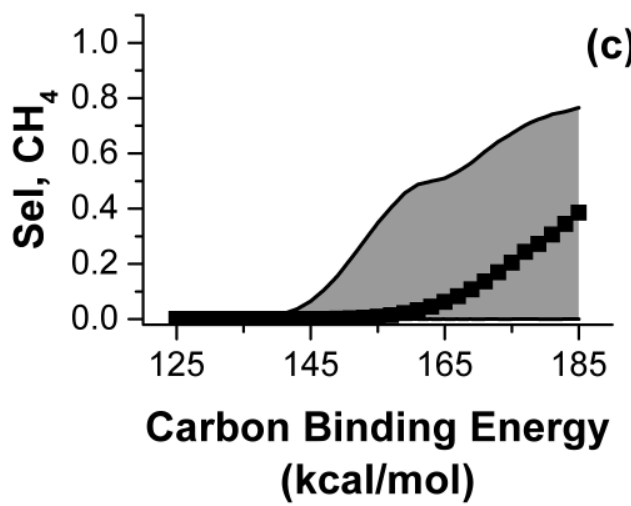

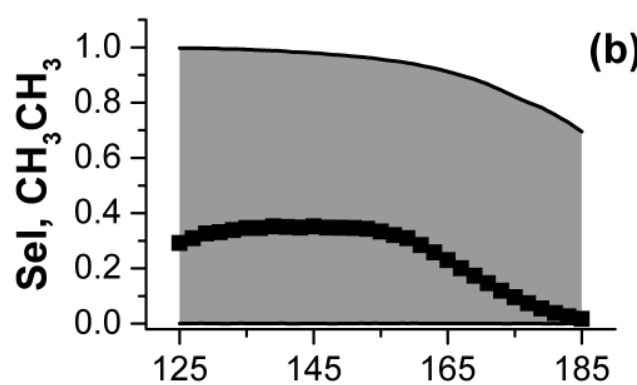

Carbon Binding Energy (kcal/mol)

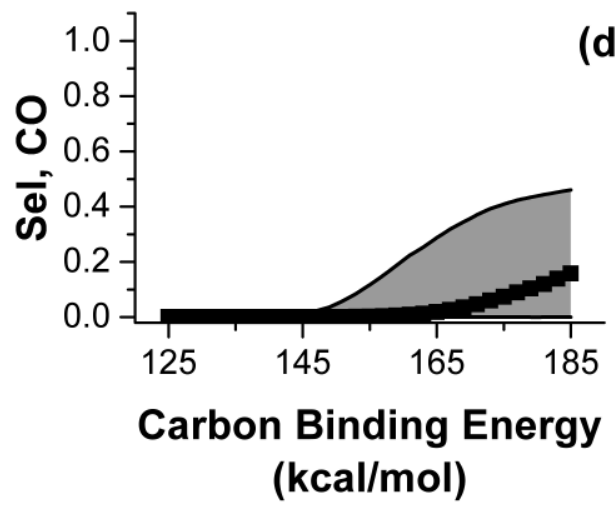

Figure 5. Uncertainty distributions in fractional carbon selectivity for major products as a function of carbon binding energy at an oxygen binding energy of $130 \mathrm{kcal} / \mathrm{mol}$. Black lines with symbols are the median $\left(50^{\text {th }}\right.$ percentile) responses, and solid black lines delineate the shaded $\pm 34 \%$ envelope (i.e., $68 \%$ of all responses are within these bounds). For a normal distribution, these percentiles are the mean \pm 1 s.d.

\section{Analysis of Mechanism and Apparent Kinetics at Representative Binding Energies}

Finally, we apply our UQ and GSA methods to gaining a more detailed mechanistic understanding of the ethanol HDO process. We specifically wish to understand the key differentiating factor between C-C and C-O cracking. To do this, we employ Stochastic Reaction Path Analysis, variance-based GSA, and UQ on the apparent kinetic parameters. 


\section{(a)}

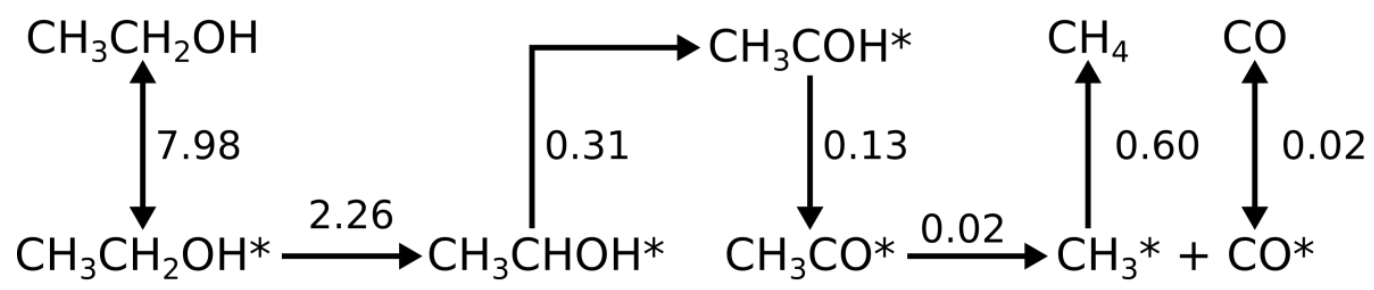

\section{(b)}

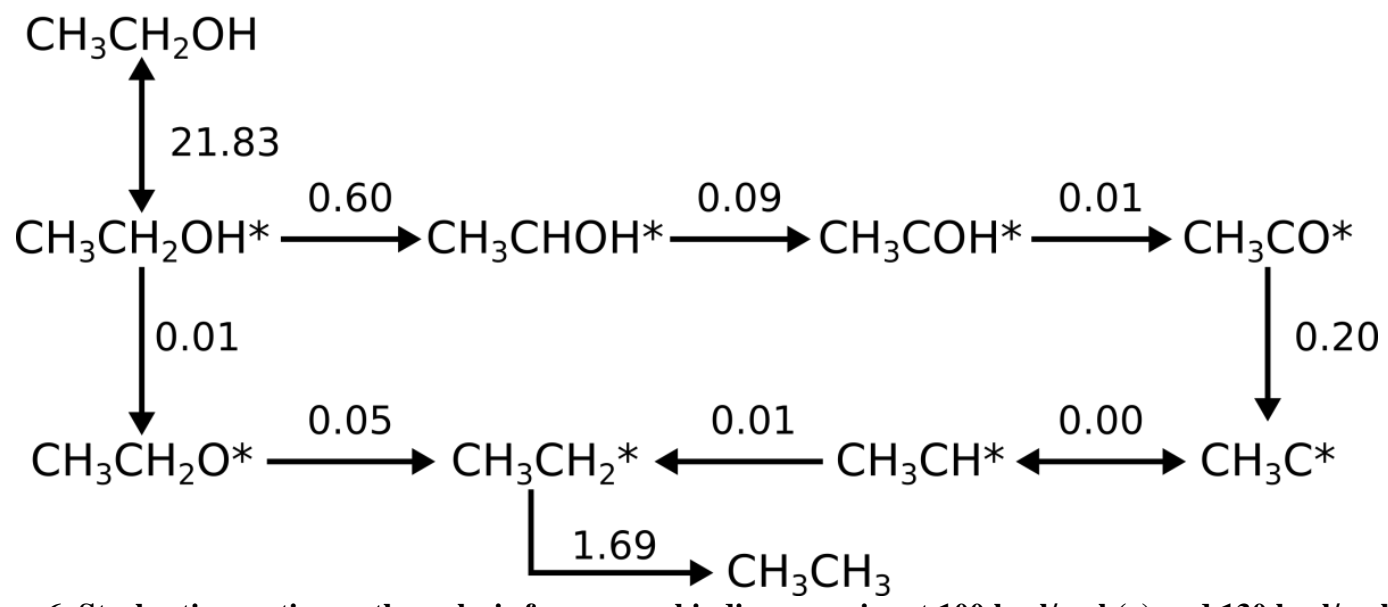

Figure 6. Stochastic reaction path analysis for oxygen binding energies at $100 \mathrm{kcal} / \mathrm{mol}$ (a) and $130 \mathrm{kcal} / \mathrm{mol}(\mathrm{b})$, both at a carbon binding energy of $155 \mathrm{kcal} / \mathrm{mol}$ (i.e., the same binding energies as in Figure 7). Single (double) arrows denote a reversible (irreversible) reaction, as determined by the median partial equilibrium index (PEI). Numbers are median net rates (in $10^{-9} \mathrm{~mol} / \mathrm{s}$ ). Uncertainty ranges for the net rates are provided in the Supporting Information. Because the distributions of the net rates for individual reactions can be quite different, the median net rates leading to and from a given species do not balance as is required in the base model.

In traditional reaction path analysis (RPA), the principal reaction pathways are identified by calculating the amount each reaction contributed to the production or consumption of every species. The stochastic reaction path analysis (SRPA) is a generalization of the traditional RPA to account for the fact we have an ensemble of models (a distribution arising from parametric uncertainty).[27] The goal of a SRPA is to analyze the distribution of species production and consumption rates over the entire ensemble of models. By its nature, the mass balances for each species do not close as they do in a traditional RPA. For the SRPA, we compute medians for the fractional production/consumption (a measure of how much each reaction contributes to the production or consumption of a given species), the rates of each elementary reaction, and the partial equilibrium indices (a measure of the degree of equilibration of an elementary reaction), and then we use the median values to identify the dominant reactions and intermediates. Figure 6 gives the SRPA at the two representative $\mathrm{O}$ binding energies. At the lower $\mathrm{O}$ binding energy (Figure 6a), the mechanism is simple thermal decomposition to $\mathrm{CO}$ and $\mathrm{CH}_{4}$, with $\mathrm{C}-\mathrm{C}$ cracking occurring in $\mathrm{CH}_{3} \mathrm{CO}^{*}$. Our DFT results[34] on several metals suggest C-C scission should actually happen in $\mathrm{CHCO}^{*}$. The difference is probably due to errors in either the thermodynamics (via the LSRs) or the kinetics (via the BEPs). At the higher $\mathrm{O}$ binding energy (Figure 6b), C-O scission occurs either in $\mathrm{CH}_{3} \mathrm{CO}^{*}$ (the principal route) or $\mathrm{CH}_{3} \mathrm{CH}_{2} \mathrm{O}^{*}$ (the secondary route). $\mathrm{H}^{*}$ on the surface then hydrogenates the $\mathrm{C}_{2}$ fragments to ethane, and $\mathrm{O}^{*}$ is removed as water (not shown). 


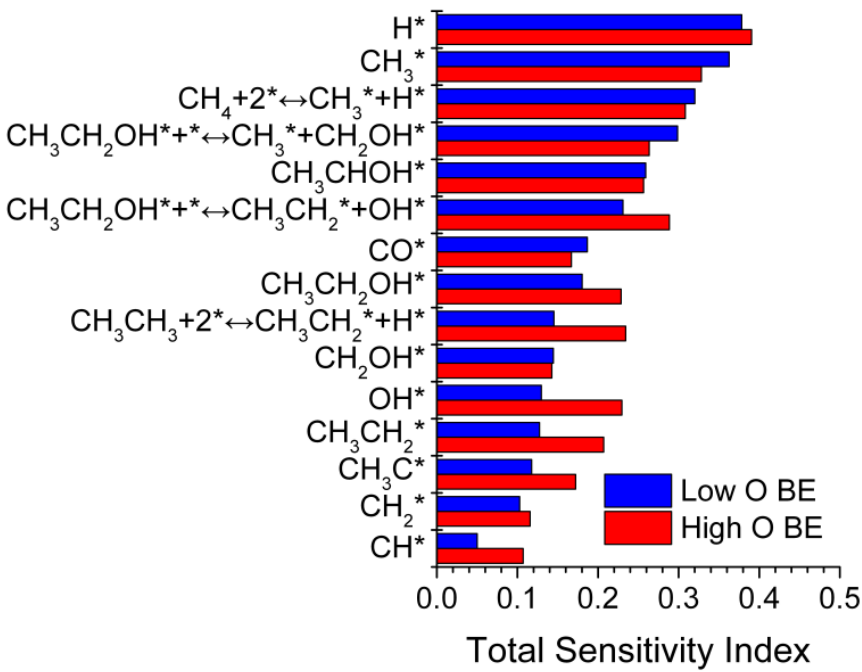

Figure 7. Total sensitivity indices on the conversion at two representative oxygen binding energies of $100 \mathrm{kcal} / \mathrm{mol}(\mathrm{low})$ and $130 \mathrm{kcal} / \mathrm{mol}$ (high) and a $\mathrm{C}$ binding energy of $155 \mathrm{kcal} / \mathrm{mol}$. Species and reactions shown are the most influential on conversion. Operating conditions are the same in Figure 1. Species energies are generally more influential than activation energies. Hydrogenations to saturated hydrocarbons, dehydroxylation and C-C bon scission from ethanol are the kinetically key reactions. The high $O$ binding energy mechanism seems to have more parametric interactions (represented by the larger number of parameters with high total sensitivity indices) than the low $O$ binding energy mechanism.

Figure 7 compares the total sensitivity indices at two representative $\mathrm{O}$ binding energies. The total sensitivity indices (see SI for mathematical definition) measure the fractional contribution of a parameter on its own and in conjunction with all other parameters to the model variance. In the absence of parametric interactions, the sum of all total sensitivity indices is unity. When the sum is larger than unity, as is the case here, parametric interactions are significant. The most important species enthalpies and reaction activation energies for both $\mathrm{O}$ binding energies are identical. The most influential parameters are related to methane formation (via $\mathrm{CH}_{\mathrm{x}}$ enthalpies and methane desorption) and $\mathrm{CO}^{*}$ desorption (controlled by the enthalpy of $\mathrm{CO}^{*}$ ), consistent with the need to remove $\mathrm{C}_{1}$ species from the surface to keep sites open for further reaction. At high $\mathrm{O}$ binding energies, the hydrogenation of $\mathrm{C}_{2}$ species and $\mathrm{OH}^{*}$ play a similar role. The remaining parameters all involve ethanol and its immediate products $\left(\mathrm{CH}_{3} \mathrm{CHOH}^{*}\right.$ and the $\mathrm{C}-\mathrm{C}$ and $\mathrm{C}-\mathrm{O}$ cracking products). Although GSA calculations routinely activate new mechanisms during the global parameter search, the activation energies of the $\mathrm{C}-\mathrm{C}$ and $\mathrm{C}-\mathrm{O}$ cracking reactions in ethanol are surprisingly influential, even though they do not occur either in the SRPA or the nominal model. We believe the reason for this is twofold. First, while the DFT activation energies for these reactions are high enough (more than $1.5 \mathrm{eV}$ ) that they would not ordinarily be expected to be active, the BEPs significantly underpredict them (sometimes by as much as $1 \mathrm{eV}$ ). Second, with the consistently underpredicted activation energy, there is a high probability to achieve an even smaller activation energy via random perturbations, and a new reaction channel opens up, enhancing the total rate. Overall, the similarity of the influential parameters underscores the common mechanism (namely thermal decomposition) present at both binding energies. Where the sensitive parameters differ is after acetyl $\left(\mathrm{CH}_{3} \mathrm{CO} *\right)$. At the lower $\mathrm{O}$ binding energy, the key parameters are related to $\mathrm{C}-\mathrm{C}$ cracking and removal of the resulting products (i.e., $\mathrm{C}_{1}$ species), whereas at the higher $\mathrm{O}$ binding energy, they are related to $\mathrm{C}-\mathrm{O}$ cracking and its resulting products $\left(\mathrm{C}_{2}\right.$ species and water).

Importantly, a larger number of species enthalpies than reaction barriers are important ( $\sim 50$ perturbed species and $\sim 300$ perturbed activation energies, resulting in 11 important species and 4 important reactions), indicating that ethanol decomposition is largely a thermochemistry-controlled process. It also points to the importance of computing and assessing experimentally accurate thermochemistry of select 
species. Importantly, the model can guide experiments, and databases of such information can be developed and shared among mechanisms. For example, the thermochemistry of $\mathrm{H}^{*}, \mathrm{OH}^{*}, \mathrm{CO}^{*}, \mathrm{H}_{2} \mathrm{O}^{*}$ is important (Figure 7); these species are common to numerous reaction mechanisms. Similarly, the $\mathrm{CH}_{\mathrm{x}}{ }^{*}$ $(\mathrm{x}=1-3)$ species are also encountered in several hydrocarbon chemistries.

The relatively large number of parameters with moderate or high total sensitivity indices indicates that parametric interactions are very important. This is underscored by the very small values of the first order sensitivity indices (the maximum value is around 0.03, see SI). Physically, this makes sense. The activity will increase the most when related parameters are varied together. For instance, the enthalpy of $\mathrm{H}^{*}$ is very influential because $\mathrm{H}^{*}$ participates in the majority of the mechanism. Furthermore, the enthalpy of $\mathrm{CH}_{3} *$ and the activation energy of $\mathrm{CH}_{3} *$ hydrogenation are also important. These three parameters $\left(\mathrm{H}^{*}\right.$ and $\mathrm{CH}_{3} *$ enthalpy and $\mathrm{CH}_{3} *$ hydrogenation activation energy) are closely connected. Increasing the enthalpy of $\mathrm{H}^{*}$ and $\mathrm{CH}_{3}{ }^{*}$ together will shift the reaction towards methane, as will lowering the activation energy of the reaction. And obviously, if these changes are made together, the rate of methane formation will increase by more than altering each of them individually, owing to the nonlinear form of the reaction rate expression. The same observation holds for each of the sensitive activation energies; one also finds that if an activation energy is sensitive, then the enthalpies of the associated adsorbates are also sensitive (note that we assumed the gas-phase species enthalpies were exact, and we did not perturb them).

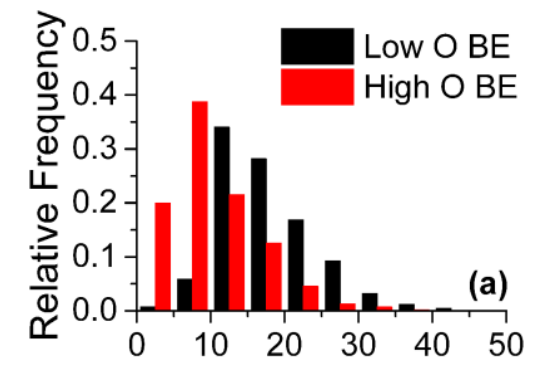

Activation Energy $(\mathrm{kcal} / \mathrm{mol})$

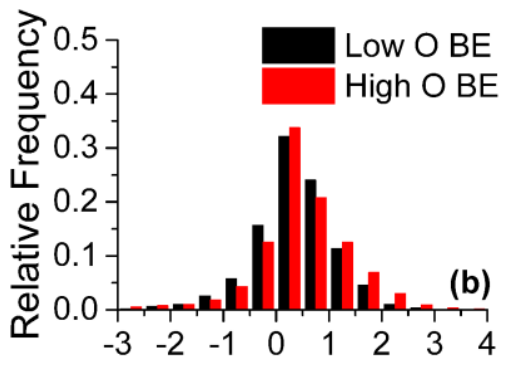

EtOH Reaction Order

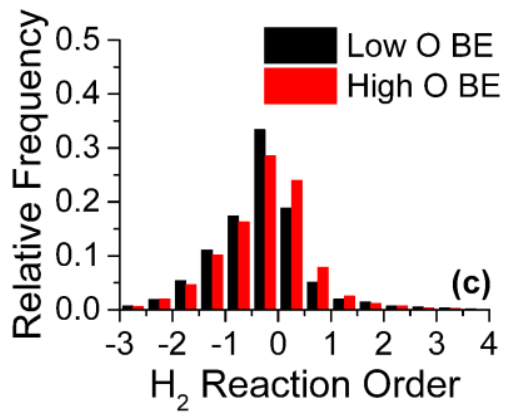

Figure 8. Uncertainty quantification histograms of apparent kinetics: activation energy (a), ethanol reaction order (b), and $\mathrm{H}_{2}$ reaction order (c). Nominal conditions are the same as in Figure 1. Temperatures are in the range $250-400{ }^{\circ} \mathrm{C}$, ethanol concentrations are in the range $1.25 \mathrm{~mol} \%-25 \mathrm{~mol} \%$, and $\mathrm{H}_{2}$ concentrations are in the range $3.75 \mathrm{~mol} \%-75$ $\mathrm{mol} \%$. C binding energy is $155 \mathrm{kcal} / \mathrm{mol}$. O binding energies (BE) are $100 \mathrm{kcal} / \mathrm{mol}$ and $130 \mathrm{kcal} / \mathrm{mol}$ (labeled as low and high, respectively).

We are also interested in the distributions of the apparent kinetic parameters, specifically the apparent activation energy and the apparent ethanol and hydrogen reaction orders. We generate distributions of the apparent kinetic parameters by quantifying the uncertainty in the rate at a series of feed conditions (temperature and composition) with the same set of sampled parameter perturbations for each feed condition. Apparent kinetic parameters are then fit for each set of rates (one set per perturbation). From the raw set of sampled apparent kinetic parameters, a distribution can be constructed. We represent these as histograms of relative frequencies as a function of the activation energy or reaction order. Relative frequencies are calculated by counting the number of times an activation energy or reaction order falls within a specified parameter range (or bin) and normalizing by the total number of sample points. The most likely activation energies (i.e., those with the highest relative frequency) are around $10 \mathrm{kcal} / \mathrm{mol}$, with the higher $\mathrm{O}$ binding energy model having a slightly lower activation energy, consistent with its inherently higher activity. In both cases, the upper bound on the activation energy is around $20 \mathrm{kcal} / \mathrm{mol}$. The higher activity at the higher $\mathrm{O}$ binding energy arises from the stronger binding of the terminal $\mathrm{O}$ in ethanol (the $\mathrm{C}$ binding energy is the same in both histograms).

The ethanol reaction orders are nearly identical and positive with most values falling between zero and one, indicating that both mechanisms share a common initial path of thermal decomposition. In both cases, there are also a large fraction of states with negative reaction orders. These negative reaction orders 
most likely are the result of combined strongly-bound species blocking free sites, which are required for decomposition to occur, with high decomposition barriers, making removal of such species slow. The total number of parameter sets with negative orders depends strongly on the $\mathrm{O}$ binding energy. At the lower $\mathrm{O}$ binding energy, the $\mathrm{H}_{2}$ order is strongly negative (most values are less than zero, with only a few slightly larger than zero). Consistent with the already high $\mathrm{H}^{*}$ coverage (see Figure 1), additional $\mathrm{H}^{*}$ blocks sites as well as reduces the driving force to dehydrogenation of ethanol. At the higher O binding energy, additional $\mathrm{H}_{2}$ has a slightly more positive order. This is consistent with the additional $\mathrm{H}^{*}$ acting both to inhibit ethanol dehydrogenation as well as removing $\mathrm{O}$ (as water), which tends to produce free sites necessary for the conversion of ethanol. These results also indicate that a change in apparent reaction orders among materials may occur. In comparing the apparent reaction orders to experimental data, one should keep in mind that idealized materials may behave differently from real materials. For example, the catalyst support or promoter may be effective at providing an alternate path for removing products from the active phase of the catalyst, resulting in free active sites and the reduction or elimination of site blocking that leads to negative reaction orders. Qualitative differences from experimental data underscores potential limitations and areas for improvement of the model.

\section{Conclusions}

We introduced the first-of-its kind uncertainty quantification (UQ) and global sensitivity analysis (GSA) to gain insights into the impact of errors of linear scaling relations (LSRs) and Bronsted-EvansPolanyi (BEP) correlatons on the predictive ability of high throughput microkinetic models. We have applied the methodology to ethanol hydrodeoxygenation (HDO). We show that the activity and selectivity depend strongly on $\mathrm{C}$ and $\mathrm{O}$ binding energies, in qualitatiave agreement with previous models on HDO of oxygenates. We find that common single metals lie in the region of simple thermal decomposition to $\mathrm{C}_{1}$ products $\left(\mathrm{CO}\right.$ and $\left.\mathrm{CH}_{4}\right)$, with the exception of $\mathrm{Ru}$, which is near the transition between $\mathrm{C}_{1}$ and $\mathrm{C}_{2}$ products (e.g., $\mathrm{C}_{2} \mathrm{H}_{6}$ ). More oxophilic materials, e.g., $\mathrm{WC}$ and $\mathrm{Mo}_{2} \mathrm{C}$, are needed for $\mathrm{HDO}$ to hydrocarbons; yet, the activity of such materials will be inherently low compared to the best materials for thermal decomposition. Interestingly, the preference towards $C_{1}$ vs. $C_{2}$ products is driven primarily by thermochemistry of adsorbates rather than kinetics. By applying UQ and GSA methods, we were also able to show that ethanol HDO has a common dehydrogenation mechanism leading to the formation of $\mathrm{CH}_{3} \mathrm{CO}^{*}$. The selectivity to $\mathrm{C}_{1}$ vs. $\mathrm{C}_{2}$ products is controlled primarily by the stability of the products of the decomposition of $\mathrm{CH}_{3} \mathrm{CO}^{*}$.

Uncertainties in the LSRs employed for estimating adsorbate enthalpies, rather than BEPs, are the dominant contributor to uncertainty in model predictions. The location of peak conversion can be estimated to within about $10 \mathrm{kcal} / \mathrm{mol}$ with current methods. This uncertainty is less than $10 \%$ of the optimal properties, i.e., the error in predictions is relatively low. Materials properties for selectivities are very robust. In contrast, uncertainties in model rates can differ up to 2-3 orders of magnitude from the median. This high uncertainty is consistent with but higher that that in recent work $[26,27]$ assessing the role of DFT errors on the rate in a DFT-based microkinetic model. As mentioned earlier, even the best available DFT functionals still show systematic errors with respect to experiments. These systematic errors will propagate to materials predictions, and improved DFT methods should be able to minimize this effect. Due to errors in the LSRs, the medians do not always closely track the nominal rate. By comparison, uncertainties in rates are less than one order of magnitude when only BEP error is accounted for.

Overall, despite the uncertainty in parameter estimation using LSRs and BEPs, the models are sufficient to guide materials selection. More accurate LSRs are needed to reduce model uncertainty, especially absolute values of rates and selectivities. In contrast, current BEP methods appear to introduce relatively little uncertainty into model predictions and very little systematic error. As a long term need, we propose to develop more accurate LSRs. As a short term alternative, one could use the existing BEPs and supply DFT-based thermochemistry for certain materials to refine specific points on these maps. Given the much lower cost of computing thermochemistry than barriers this may be a reasonbale compromise. 
An even more appealing approach we propose is to employ hierarchical refinement, i.e., predict maps using LSRs and BEPs and refine the important thermochemistry only (obtained via LSRs) using DFT. This is a very doable task.

\section{Supporting Information}

Supporting Information on DFT calculations (including total energies and images of converged structures), BEP correlations, variance-based global sensitivity analysis, and UQ maps for the location of maximum conversion in the presence of LSR only errors is available.

\section{Acknowledgments}

The ethanol mechanism and the DFT calculations were supported from the Catalysis Center for Energy Innovation (CCEI), an Energy Frontier Research Center funded by the U.S. Department of Energy, Office of Science, Office of Basic Energy Sciences under award number DE-SC0001004. The uncertainty analysis was supported by the U.S.Department of Energy Office of Science, Office of Advanced Scientific Computing Research, and Applied Mathematics program under Award No. DESC0010549.The authors gratefully acknowledge computer time from the Center for Functional Nanomaterials, Brookhaven National Laboratory, supported by the U.S. Department of Energy, Office of Basic Energy Sciences under Contract No. DE-AC02-98CH10886.

\section{References}

[1] D. Farrusseng, High-throughput heterogeneous catalysis, Surface Science Reports, 63 (2008) 487-513.

[2] M. Salciccioli, M. Stamatakis, S. Caratzoulas, D.G. Vlachos, A review of multiscale modeling of metalcatalyzed reactions: Mechanism development for complexity and emergent behavior, Chemical Engineering Science, 66 (2011) 4319-4355.

[3] A. Logadottir, T. Rod, J. Norskov, B. Hammer, S. Dahl, C. Jacobsen, The Bronsted-Evans-Polanyi relation and the volcano plot for ammonia synthesis over transition metal catalysts, Journal Of Catalysis, 197 (2001) 229-231.

[4] Z. Ulissi, V. Prasad, D.G. Vlachos, Effect of multiscale model uncertainty on identification of optimal catalyst properties, Journal Of Catalysis, 281 (2011) 339-344.

[5] T. Bligaard, J. Norskov, S. Dahl, J. Matthiesen, C. Christensen, J. Sehested, The Bronsted-EvansPolanyi relation and the volcano curve in heterogeneous catalysis, Journal Of Catalysis, 224 (2004) 206217.

[6] M. Andersson, T. Bligaard, A. Kustov, K. Larsen, J. Greeley, T. Johannessen, C. Christensen, J. Norskov, Toward computational screening in heterogeneous catalysis: Pareto-optimal methanation catalysts, Journal of Catalysis, 239 (2006) 501-506.

[7] J. Cheng, P. Hu, Utilization of the three-dimensional volcano surface to understand the chemistry of multiphase systems in heterogeneous catalysis., J. Am. Chem. Soc., 130 (2008) 10868-10869.

[8] G. Jones, J. Jakobsen, S. Shim, J. Kleis, M. Andersson, J. Rossmeisl, F. Abild-pedersen, T. Bligaard, S. Helveg, B. Hinnemann, J. Rostrup-nielsen, I. Chorkendorff, J. Sehested, J. Norskov, First principles calculations and experimental insight into methane steam reforming over transition metal catalysts, Journal Of Catalysis, 259 (2008) 147-160.

[9] J. Greeley, J. Norskov, Combinatorial Density Functional Theory-Based Screening of Surface Alloys for the Oxygen Reduction Reaction, Journal Of Physical Chemistry C, 113 (2009) 4932-4939.

[10] P. Ferrin, D. Simonetti, S. Kandoi, E. Kunkes, J. Dumesic, J. Norskov, M. Mavrikakis, Modeling Ethanol Decomposition on Transition Metals: A Combined Application of Scaling and Bronsted-EvansPolanyi Relations, Journal Of The American Chemical Society, 131 (2009) 5809-5815. 
[11] M. Salciccioli, D.G. Vlachos, Kinetic Modeling of Pt Catalyzed and Computation-Driven Catalyst Discovery for Ethylene Glycol Decomposition, ACS Catalysis, 1 (2011) 1246-1256.

[12] A.J. Medford, A. Vojvodic, J.S. Hummelshøj, J. Voss, F. Abild-Pedersen, F. Studt, T. Bligaard, A. Nilsson, J.K. Nørskov, From the Sabatier principle to a predictive theory of transition-metal heterogeneous catalysis, Journal Of Catalysis, 328 (2015) 36-42.

[13] F. Abild-Pedersen, J. Greeley, F. Studt, J. Rossmeisl, T.R. Munter, P.G. Moses, E. Skulason, T. Bligaard, J.K. Norskov, Scaling properties of adsorption energies for hydrogen-containing molecules on transition-metal surfaces, Phys. Rev. Lett., 99 (2007) 016105.

[14] E.M. Fernández, P.G. Moses, A. Toftelund, H.A. Hansen, J.I. Martínez, F. Abild-Pedersen, J. Kleis, B. Hinnemann, J. Rossmeisl, T. Bligaard, J.K. Nørskov, Scaling relationships for adsorption energies on transition metal oxide, sulfide, and nitride surfaces., Angew. Chem. Int. Ed. Engl., 47 (2008) 4683-4686.

[15] M. Salciccioli, Y. Chen, D. Vlachos, Density Functional Theory-Derived Group Additivity and Linear Scaling Methods for Prediction of Oxygenate Stability on Metal Catalysts: Adsorption of Open-Ring Alcohol and Polyol Dehydrogenation Intermediates on Pt-Based Metals, Journal Of Physical Chemistry C, 114 (2010) 20155-20166.

[16] G. Jones, F. Studt, F. Abild-Pedersen, J.K. Norskov, T. Bligaard, Scaling relationships for adsorption energies of C-2 hydrocarbons on transition metal surfaces, Chemical Engineering Science, 66 (2011) 6318-6323.

[17] J.E. Sutton, D.G. Vlachos, Error estimates in semi-empirical estimation methods of surface reactions, Journal Of Catalysis, 297 (2013) 202-216.

[18] E. Shustorovich, A. Zeigarnik, The UBI-QEP method: Basic formalism and applications to chemisorption phenomena on transition metal surfaces. Chemisorption energetics, Russian Journal Of Physical Chemistry, 80 (2006) 4-30.

[19] M. Maestri, K. Reuter, Semiempirical Rate Constants for Complex Chemical Kinetics: First-Principles Assessment and Rational Refinement, Angewandte Chemie International Edition, 50 (2011) 1194-1197.

[20] V. Pallassana, M. Neurock, Electronic factors governing ethylene hydrogenation and dehydrogenation activity of pseudomorphic Pd-ML/Re(0001), Pd-ML/Ru(0001), Pd(111), and Pd$\mathrm{ML/Au(111)} \mathrm{surfaces,} \mathrm{Journal} \mathrm{of} \mathrm{Catalysis,} 191$ (2000) 301-317.

[21] R. Alcala, M. Mavrikakis, J. Dumesic, DFT studies for cleavage of C-C and C-O bonds in surface species derived from ethanol on Pt(111), Journal of Catalysis, 218 (2003) 178-190.

[22] A. Michaelides, Z. Liu, C. Zhang, A. Alavi, D. King, P. Hu, Identification of general linear relationships between activation energies and enthalpy changes for dissociation reactions at surfaces, Journal Of The American Chemical Society, 125 (2003) 3704-3705.

[23] R. Van SANTEN, W. OFFERMANS, K. MALEK, E. PIDKO, Computational modeling of catalytic reactivity, Molecular Simulation, 33 (2007) 327-336.

[24] J.E. Sutton, D.G. Vlachos, A Theoretical and Computational Analysis of Linear Free Energy Relations for the Estimation of Activation Energies, ACS Catalysis, 2 (2012) 1624-1634.

[25] J.E. Sutton, D.G. Vlachos, Building large microkinetic models with first-principles' accuracy at reduced computational cost, Chemical Engineering Science, 121 (2015) 190-199.

[26] A.J. Medford, J. Wellendorff, A. Vojvodic, F. Studt, F. Abild-Pedersen, K.W. Jacobsen, T. Bligaard, J.K. Nørskov, Assessing the reliability of calculated catalytic ammonia synthesis rates, Science, 345 (2014) 197-200.

[27] J.E. Sutton, W. Guo, M.A. Katsoulakis, D.G. Vlachos, Effects of correlated parameters and uncertainty in electronic-structure-based chemical kinetic modelling, Nature Chemistry, (2016).

[28] D. Loffreda, F.c. Delbecq, F. Vigné, P. Sautet, Fast prediction of selectivity in heterogeneous catalysis from extended Brønsted-Evans-Polanyi relations: a theoretical insight., Angew. Chem. Int. Ed. Engl., 48 (2009) 8978-8980. 
[29] J. Zaffran, C. Michel, F. Auneau, F. Delbecq, P. Sautet, Linear Energy Relations As Predictive Tools for Polyalcohol Catalytic Reactivity, Acs Catalysis, 4 (2014) 464-468.

[30] J. Zaffran, C. Michel, F. Delbecq, P. Sautet, Trade-Off between Accuracy and Universality in Linear Energy Relations for Alcohol Dehydrogenation on Transition Metals, The Journal of Physical Chemistry C, 119 (2015) 12988-12998.

[31] S. Wang, V. Petzold, V. Tripkovic, J. Kleis, J.G. Howalt, E. Skúlason, E.M. Fernández, B. Hvolb\aek, G. Jones, A. Toftelund, H. Falsig, M. Björketun, F. Studt, F. Abild-Pedersen, J. Rossmeisl, J.K. Nørskov, T. Bligaard, Universal transition state scaling relations for (de)hydrogenation over transition metals., Phys Chem Chem Phys, 13 (2011) 20760-20765.

[32] A. Mhadeshwar, D. Vlachos, Hierarchical, multiscale surface reaction mechanism development: CO and $\mathrm{H}-2$ oxidation, water-gas shift, and preferential oxidation of $\mathrm{CO}$ on Rh, Journal of Catalysis, 234 (2005) 48-63.

[33] J. Soler, E. Artacho, J. Gale, A. García, J. Junquera, P. Ordejón, D. Sánchez-Portal, The SIESTA method for ab initio order- $N$ materials simulation, J Phys: Condens Matter, 14 (2002) 2745-2779.

[34] J.E. Sutton, D.G. Vlachos, Ethanol Activation on Closed-Packed Surfaces, INDUSTRIAL \& ENGINEERING CHEMISTRY RESEARCH, 54 (2015) 4213-4225.

[35] J.P. Perdew, K. Burke, M. Ernzerhof, Generalized gradient approximation made simple, Physical Review Letters, 77 (1996) 3865-3868.

[36] J. Wellendorff, T.L. Silbaugh, D. Garcia-Pintos, J.K. Nørskov, T. Bligaard, F. Studt, C.T. Campbell, A benchmark database for adsorption bond energies to transition metal surfaces and comparison to selected DFT functionals, Surface Science, 640 (2015) 36-44.

[37] A. Alavi, P. Hu, T. Deutsch, P.L. Silvestrelli, J. Hutter, CO Oxidation on Pt(111): An Ab Initio Density Functional Theory Study, Physical Review Letters, 80 (1998) 3650-3653.

[38] H. Wang, Z. Liu, Comprehensive mechanism and structure-sensitivity of ethanol oxidation on platinum: New transition-state searching method for resolving the complex reaction network, Journal Of The American Chemical Society, 130 (2008) 10996-11004.

[39] J.E. Sutton, P. Panagiotopoulou, X.E. Verykios, D.G. Vlachos, Combined DFT, Microkinetic, and Experimental Study of Ethanol Steam Reforming on Pt, The Journal of Physical Chemistry C, 117 (2013) 4691-4706.

[40] S. Wang, V. Vorotnikov, J.E. Sutton, D.G. Vlachos, Bronsted-Evans-Polanyi and Transition State Scaling Relations of Furan Derivatives on Pd(111) and Their Relation to Those of Small Molecules, Acs Catalysis, 4 (2014) 604-612.

[41] A.B. Mhadeshwar, H. Wang, D.G. Vlachos, Thermodynamic consistency in microkinetic development of surface reaction mechanisms, Journal of Physical Chemistry B, 107 (2003) 12721-12733.

[42] I.M. Sobol, Global sensitivity indices for nonlinear mathematical models and their Monte Carlo estimates, Math. Comput. Simul., 55 (2001) 271-280.

[43] A. Saltelli, Making best use of model evaluations to compute sensitivity indices, Computer Physics Communications, 145 (2002) 280-297.

[44] A. Saltelli, P. Annoni, I. Azzini, F. Campolongo, M. Ratto, S. Tarantola, Variance based sensitivity analysis of model output. Design and estimator for the total sensitivity index, Computer Physics Communications, 181 (2010) 259-270.

[45] F. Campolongo, A. Saltelli, J. Cariboni, From screening to quantitative sensitivity analysis. A unified approach, Computer Physics Communications, 182 (2011) 978-988.

[46] A. Saltelli, M. Ratto, S. Tarantola, F. Campolongo, Update 1 of: Sensitivity Analysis for Chemical Models, CHEMICAL REVIEWS, 112 (2012) PR1-PR21.

[47] J. Jae, W. Zheng, R.F. Lobo, D.G. Vlachos, Production of Dimethylfuran from Hydroxymethylfurfural through Catalytic Transfer Hydrogenation with Ruthenium Supported on Carbon, ChemSusChem, 6 (2013) 1158-1162. 



\title{
Effect of Errors in Linear Scaling Relations and Brønsted-Evans-Polanyi Relations on Activity and Selectivity Maps
}

\author{
Jonathan E. Sutton and Dionisios G. Vlachos* \\ Catalysis Center for Energy Innovation and Center for Catalytic Science and Technology, Department \\ of Chemical and Biomolecular Engineering, University of Delaware, Newark, DE 19716
}

\begin{abstract}
We introduce for the first time uncertainty quantification and global sensitivity analysis to assess the effect of error in linear scaling relations (LSRs) and Brønsted-Evans-Polanyi (BEP) relations on activity and selectivity maps of microkinetic models, which have recently been used for in silico prediction of new materials. The method is applied to ethanol hydrodeoxygenation (HDO). Selectivity trends are driven by adsorbate thermochemistry rather than kinetics. Uncertainty quantification calculations show that the most likely location of the maximum conversion can be estimated to within about $10 \mathrm{kcal} / \mathrm{mol}$ in the $\mathrm{C}$ and $\mathrm{O}$ binding energies. Broad selectivity trends are even more robust. Model rates show uncertainties of 2-3 orders of magnitude about the median. Uncertainty in the activity predictions is dominated by that of the LSRs. Our calculations demonstrate that there is a common initial mechanism of ethanol HDO and decomposition, and the stability of decomposition products is crucial to determining the selectivity.
\end{abstract}

Keywords: Ethanol, hydrodeoxygenation, density functional theory, microkinetic modeling, uncertainty quantification, sensitivity analysis, Linear Scaling Relations, Brønsted-Evans-Polanyi correlations

*Corresponding author: e-mail: vlachos@udel.edu; tel. 302-831-2830. 


\section{Introduction}

Model-aided catalyst prediction has been a major topic of research over the last two decades[1,2] due to the promise of developing improved and/or lower cost catalysts and of eliminating intensive empirical testing. To this end, high throughput microkinetic models have been developed for prototypical processes such as ammonia synthesis[3] and decomposition[4], methanation[5, 6], CO hydrogenation[7], methane steam reforming[8], oxygen reduction[9], ethanol decomposition[10], ethylene glycol decomposition[11], and methanol and higher alcohol synthesis.[12]

In the quest of searching for new materials, upon constructing a mechanism, kinetic parameters are estimated without resorting to expensive parameter estimation methods (e.g., density functional theory (DFT)). In practice, atomic binding energies (descriptors) are mapped to species energies via linear scaling relations [13-17] (LSRs) or bond order conservation (BOC) [18, 19] and species energies (or heats of reactions, which are their linear combinations, to be more precise) are mapped to reaction activation energies via Brønsted-Evans-Polanyi relations[5, 10, 11, 20-24] (BEPs). These correlations have recently been referred to as first principles-based semi-empirical methods (FPSEM) or first-principles-based screening methods,[25] and due to their simple linear functional form, they are capable of estimating an entire set of kinetic parameters in real time. We term microkinetic (MKM) models using this approach as descriptor-based (in contrast to first-principles-based MKM). The combination of LSRs and BEPs enables one to estimate thermochemistry and activation energies of large reaction networks or for arbitrary materials. The former is essential for modeling complex reaction networks and the latter enables construction of activity and selectivity maps. We provide an example below. These maps in turn elucidate the optimal material properties that could lead to discovery of new catalysts.

These correlations are generally regressed from DFT data, and the regression process invariably introduces additional error above and beyond what is inherently present in the underlying DFT energies (this topic has recently been addressed by Nørskov and coworkers[26] as well as by us[27]). We have previously quantified this error and investigated how it propagates when various FPSEM correlations are combined, [17] and Maestri and Reuter have previously investigated the accuracy of BOC estimates.[19] Further analysis of BEP accuracy has been carried out by Sautet and coworkers.[28-30] While Ulissi et al. have considered the impact of uncertainty in BOC on predictions of ammonia decomposition catalysts with maximum activity,[4] to our knowledge, no prior work has been done on evaluating the impact of errors in LSR and BEP correlations on MKM uncertainty. Total model uncertainty (once ascertained) could be reduced by identifying the most sensitive parameters via a sensitivity analysis and by refining them with improved estimates, in a procedure known as hierarchical refinement.[25] We are unaware of any studies on identifying the most influential parameters in the presence of parametric uncertainty.

In this work, we address these two topics. We first outline the process of developing a high throughput MKM and draw preliminary conclusions into the important parameters and the mechanism. We do this with a model of ethanol hydrodeoxygenation (HDO) as a surrogate that might be used to upgrade biomass into value-added fuels and chemicals. Then we employ uncertainty quantification (UQ) methods to assess the overall contributions of the LSRs and BEPs individually and in combination to the total uncertainty in (1) the 'location' of the maximum conversion and selectivity, i.e., the atomic binding energies and (2) in conversion and selectivity as a function of atomic binding energies. This UQ is carried out with respect to the original model predictions, rather than with respect to experiments. Finally, we employ stochastic reaction path analysis,[27] uncertainty quantification and variance-based global sensitivity analysis (GSA), which explicitly incorporates parametric uncertainty, to explore the mechanism of ethanol HDO at representative catalyst formulations.

\section{Methods}

\section{Density Functional Theory Calculations}

Select DFT calculations were carried out in order to parameterize BEP relations for reactions in the water-gas mechanism and for the oxidative dehydrogenation of terminal $\mathrm{OH}$ groups by coadsorbed $\mathrm{O} *$ or 
$\mathrm{OH}^{*}$ on the close-packed facets of $\mathrm{Co}, \mathrm{Ni}, \mathrm{Pd}, \mathrm{Pt}, \mathrm{Rh}$, and $\mathrm{Ru}$. The results of the DFT calculations and the BEP correlations are given in the Supporting Information (SI). We have chosen to employ the closepacked facets rather than other, more open facets (e.g., steps or open terraces) that are often the active phase to reduce the number of DFT calculations. This simplification should not alter our general conclusions because LSR and BEP correlations for terraces and steps are typically similar, with slopes and intercepts that differ by a small amount.[13, 31] Furthermore, by scanning over a wide range of atomic binding energies, we implicitly include the more open facets, although with some systematic error. Overall, we believe that close-packed facets provide a reasonable starting point for a qualitative identification of promising materials. More quantitative predictions must be made with higher fidelity models, such as kinetic Monte Carlo simulations, of various facets. One can view this an implementation of the principle of hierarchical model refinement.[25, 32]

DFT calculations were performed with the SIESTA code.[33] Computational parameters followed a method employed in previous work.[34] We used 3x3 unit cells with four metal layers (bottom two layers frozen) and $15 \AA$ vacuum slabs with a 3x3x1 Monkhorst-Pack k-point mesh and the PBE functional.[35] This functional tends to result in higher binding energies compared to the best available experimental data.[36] The systematic errors in this functional (note that systematic errors are common to all the GGAtype functionals) will result in systematic errors in model predictions with respect to experiment. Geometries were considered relaxed when a force tolerance of $0.1 \mathrm{eV} / \AA$ was reached. This force tolerance was chosen to reduce the computational burden associated with the large number of calculations required for training the semi-empirical correlations. Our structures obtained with this tolerance closely match the structures obtained with a more rigorous force tolerance $(0.05 \mathrm{eV} / \AA)$ and finer k-point mesh $(5 \times 5 \times 1$ for the same supercell).[15] We expect that this similarity extends to the energies as well and errors in the energies arising from the less rigorous force tolerance will be smaller than the errors arising from the correlations fit to the training data. Transition states were located via a constrained optimization technique[37] coupled with an automatic transition state search algorithm.[38] Prior work has shown that this procedure yields reasonable estimates of the transition state geometries that are sufficient for parameterizing the BEP relations required for this work.

\section{Microkinetic Modeling (MKM)}

The high throughput MKM of ethanol HDO in this work was based on our prior model of ethanol steam reforming on Pt.[25, 39] To our earlier model we added oxidative dehydrogenation reactions for the removal of $\mathrm{H}$ by coadsorbed $\mathrm{O}^{*}$ or $\mathrm{OH}^{*}$, i.e., $\mathrm{RH}^{*}+\mathrm{O}^{*}=\mathrm{R}^{*}+\mathrm{OH}^{*}$ and $\mathrm{RH}^{*}+\mathrm{OH}^{*}=\mathrm{R}^{*}+\mathrm{OH}_{2} *$, where $\mathrm{R}$ is any intermediate of ethanol in proximity with the surface to enable $\mathrm{H}$ transfer. In total, our revised mechanism has 67 species and 300 reactions.

To rapidly parameterize the microkinetic model, two types of semi-empirical correlations were employed: extended LSRs (ELSRs) for species energies and BEP relations for activation energies. These correlations are obtained by performing DFT calculations for a wide variety of intermediates and transition states and then fitting correlations to the training data. Correlation errors are estimated from the deviations between correlation estimates and the corresponding DFT data.

Species energies were estimated from carbon and oxygen atomic binding energies via ELSRs, with parameters taken from our prior work.[17] The ELSRs have the following functional form (adapted from Eq. 25, Ref. [17]):

$$
\hat{E}_{M_{2}}=\hat{E}_{M_{1}}+\sum_{j} \sum_{i} \gamma_{j}\left(E_{i, M_{2}}-E_{i, M_{1}}\right)
$$

where $\hat{E}_{M_{2}}$ is the estimate of the binding energy on metal 2, $\hat{E}_{M_{1}}$ is the reference binding energy on metal $1, \gamma_{j}$ is the slope (obtained from regressing DFT data) approximately measuring the contribution of the metal to the electron density around heteroatom $j, E_{i, M_{2}}$ is the atomic binding energy of the type $i$ heteroatom on metal 2, and $E_{i, M_{1}}$ is its equivalent for metal 1 . The heteroatom index $j$ is for convenience, as the same heteroatom type may be repeated (e.g., two distinct $\mathrm{C}$ binding centers). The reference 
energies for the ELSRs were taken from our prior DFT-based temperature-corrected thermochemistry on $\mathrm{Pt},[25,39]$ which in turn were based on DFT calculations by Salciccioli et al.[15] Adsorbate lateral interactions were adjusted as atomic binding energies vary using the method of Salciccioli and Vlachos.[11]

Activation energies were estimated using BEP relations for activation of ethanol on a variety of transition metals. These BEP relations are a combination of prior work[34] and new calculations (see the SI). Consistent with our findings on Pt[39], oxidative dehydrogenation reactions were assumed to follow the corresponding thermal dehydrogenation homologous series, with the sole exception of the dehydrogenation of terminal $\mathrm{OH}$ groups by adsorbed $\mathrm{OH}^{*}$ (in these reactions hydrogen bonding plays a key role and barriers are very small or absent). Owing to the importance of the water-gas shift mechanism in removing adsorbed $\mathrm{O}^{*}$ and $\mathrm{OH}^{*}$ and oxidizing $\mathrm{CO}^{*}$, we also developed BEP relations specifically for the water-gas shift chemistry (see the SI). For convenience, we summarize the LSR parameters in Table 1 and the BEP parameters in Table 2.

Table 1. Extended linear scaling relations (ELSR) slopes (reference binding energies were from Pt). The overall uncertainty in the LSR estimates was estimated as $0.023 \pm 0.308 \mathrm{eV}$ (mean \pm s.d.). The water LSR slope was arbitrarily set to 0.1 (weak dependence on the metal) and was assumed to hold for closed-shell alcohols as well. LSR parameters taken from Sutton and Vlachos.[17]

\begin{tabular}{cc} 
Binding Mode & Slope \\
\hline $\mathrm{CH}_{3}$ & 0.4451 \\
$\mathrm{CH}_{2}$ & 0.5663 \\
$\mathrm{CH}$ & 0.8182 \\
$\mathrm{CH}_{2} \mathrm{OH}$ & 0.4151 \\
$\mathrm{CHOH}$ & 0.7140 \\
$\mathrm{COH}$ & 0.6586 \\
$\mathrm{CH}_{2} \mathrm{O}$ & \\
(via $\mathrm{C}$ ) & 0.2561 \\
$\mathrm{CH} \mathrm{O}_{2} \mathrm{O}$ & \\
(via $\mathrm{O}$ ) & 0.1426 \\
$\mathrm{CHO}$ & 0.6571 \\
$\mathrm{CO}$ & 0.4174 \\
$\mathrm{OH}$ & 0.3672 \\
$\mathrm{H}_{2} \mathrm{O}$ & 0.1000
\end{tabular}

Table 2. BEP parameters and standard deviations in the errors (the means were assumed to be zero).

\begin{tabular}{|c|c|c|c|c|}
\hline Homologous Series & Slope & Intercept $(\mathrm{eV})$ & Std Dev $(\mathrm{eV})$ & Reference \\
\hline C-H cleavage ${ }^{1}$ & 0.5860 & 0.7840 & 0.2882 & [34] \\
\hline O-H cleavage ${ }^{1}$ & 0.2815 & 0.7550 & 0.1454 & [34] \\
\hline C-C cleavage ${ }^{1}$ & 0.7106 & 1.3031 & 0.4138 & [34] \\
\hline $\mathrm{C}-\mathrm{OH}$ cleavage ${ }^{1}$ & 0.5801 & 1.2177 & 0.3916 & [34] \\
\hline C-O cleavage ${ }^{1}$ & 0.8125 & 1.4675 & 0.3945 & [34] \\
\hline $\mathrm{CH}_{3} \mathrm{CH}_{2} \mathrm{OH}, \alpha \mathrm{C}-\mathrm{H}^{2}$ & 0.6954 & 0.9271 & 0.0416 & [34] \\
\hline $\mathrm{CH}_{3} \mathrm{CH}_{2} \mathrm{O}-\mathrm{H}^{2}$ & 0.1872 & 0.8487 & 0.0770 & [34] \\
\hline $\mathrm{CH}_{3} \mathrm{COH}, \mathrm{O}-\mathrm{H}^{2}$ & 0.2352 & 0.7472 & 0.1206 & [34] \\
\hline $\mathrm{CH}_{3} \mathrm{C}-\mathrm{OH}^{2}$ & 0.5284 & 0.9458 & 0.1012 & [34] \\
\hline $\mathrm{CH}_{3}-\mathrm{CO}^{2}$ & 0.7794 & 1.2493 & 0.1832 & [34] \\
\hline $\mathrm{CH}_{3} \mathrm{C}-\mathrm{O}$ & 1.5016 & 1.6535 & 0.2031 & [34] \\
\hline $\mathrm{CH}_{2} \mathrm{CO}, \beta \mathrm{C}-\mathrm{H}^{2}$ & 1.1049 & 1.0611 & 0.2132 & [34] \\
\hline $\mathrm{CH}_{2}-\mathrm{CO}^{2}$ & 0.7900 & 1.1023 & 0.3225 & [34] \\
\hline
\end{tabular}




$\begin{array}{lllll}\mathrm{CH}-\mathrm{CO}^{2} & 0.5333 & 0.5815 & 0.0730 & \text { [34] } \\ \mathrm{H}_{2} \mathrm{O}, \mathrm{O}-\mathrm{H}^{2,3} & 0 & 0.8734 & 0.1015 & \text { This work } \\ \mathrm{O}-\mathrm{H}^{2,3} & 0 & 1.2311 & 0.3310 & \text { This work } \\ \mathrm{CO}+\mathrm{O}^{2,4} & 0.2816 & 0.9701 & 0.0911 & \text { This work } \\ \mathrm{CO}+\mathrm{OH}^{2} & 0.7455 & 0.6565 & 0.0505 & \text { This work } \\ \mathrm{COO}-\mathrm{H}^{2,3} & 0 & 0.8942 & 0.1221 & \text { This work } \\ \mathrm{O}-\mathrm{H} \mathrm{ODH} & & 0.3430 & 0.7342 & \text { This work }\end{array}$

1. Universal BEP for a variety of reactants on all metals.

2. Reaction-specific BEP as a function of metal.

3. Slope was not statistically significant and close to zero, so the best-fit BEP was assumed to just be the mean activation energy (slope explicitly set to zero).

4. Excludes anomalously high barrier on Pt.

The correlations in Table 1 and Table 2 were obtained via ordinary least squares minimization in order to minimize the average deviation from the training data. Generally, the correlation parameters retain their physical meaning. For instance, the ELSR slope is based on the fraction of heteroatom electron density provided by the surface, and the BEP slope is a measure of the earliness or lateness of the transition state (ranging from 0 to 1 ). Very small (0) or very large (1) BEP slopes merely imply that the transition state is identical to the reactant or product, respectively. We have previously found that BEP slopes for the same set of reactions written in opposite directions must sum to unity,[24] implying that whether a transition state is reactant-like or product-like is entirely dependent on the choice of convention for the reactant and product. Occasionally, however, the least-squares-derived parameters exceed the accepted range, potentially for two possible reasons. For small deviations (smaller than about 0.1 for BEP slopes), it is likely a reflection of the known tendency for small molecules to have more significant geometric relaxations than large molecules.[40] For larger deviations (e.g., the slope of 1.5 for $\mathrm{CH}_{3} \mathrm{CO}$ $\mathrm{C}-\mathrm{O}$ scission), the more likely source is that two or more distinct homologous series have been included in the regression. For this specific case, if the points for the non-oxophilic metals $\mathrm{Pd}$ and Pt are excluded from the points for the more oxophilic metals $\mathrm{Co}, \mathrm{Ni}, \mathrm{Rh}$, and $\mathrm{Ru}$, the slope decreases to about 1 .

This microkinetic model was then solved using a triangular mesh (to maximize the density of mesh points for a given spacing and to simplify discrete binning of points for later analysis of the distributions of the locations of maximum conversion and selectivity) of carbon and oxygen binding energies to identify trends in activity, selectivity, and adsorbate coverage. Maps were generated with a spacing of 2 $\mathrm{kcal} / \mathrm{mol}$ between mesh points for the nominal model (in the absence of uncertainty) and with a spacing of $3 \mathrm{kcal} / \mathrm{mol}$ for the UQ calculations. The coarser mesh was required to reduce the high computational burden associated with the UQ calculations.

\section{Uncertainty Quantification and Global Sensitivity Analysis}

One of the key aspects of this work is the explicit incorporation of UQ and GSA. UQ assists in assessing the inherent variability of model predictions and GSA provides quantitative insight into which parameters are the principal source of that variability.

UQ calculations were performed by assuming that errors in the LSRs and BEPs arise from the least squares regression and not from the underlying DFT data, as we expect that the linear correlation errors will have more impact on model uncertainty than the DFT errors. The deviations between least squares regression estimates and the corresponding actual values are taken to follow the same normal distribution, and individual errors are independent of each other (this also implies no correlations exist between them). Following these two standard assumptions, we sample the error in each species or reaction energy using the distributions of the deviations between the actual DFT data and the corresponding correlation estimates, i.e., using a normal distribution that describes the 'scatter' of each correlation. The statistics of the distributions are summarized in Tables 1 and 2.

The UQ and GSA methodology maintains thermodynamic consistency and proceeds as follows. We first estimate species energies with the LSRs and then add a perturbation sampled from the uncertainty distribution. This results in perturbed species energies. Reaction energies are calculated from the 
perturbed species energies and automatically incorporate LSR uncertainty. Given that species energies form a thermodynamic basis for reaction networks which consist of more reactions than species, this ensures that all pathways are thermodynamically consistent.[41] We have previously shown that BEP correlation estimates are subject to two types of error: errors related to the reaction energy inputs and errors intrinsic to the regression process.[17] For sampling the BEP error, employing the perturbed reaction energies as input to the BEP relation automatically accounts for the first source of error. The second source of error is then sampled directly, and this perturbation is added to the estimated forward activation energy. Reverse activation energies are calculated from the forward activation energies and the reaction energies. This approach ensures that the kinetics is also consistent with the thermodynamics of the entire network.[41] By applying perturbations at each stage of the parameters' estimation process, the LSR uncertainty propagates to the activation energy as described in our previous work. [17] The combined uncertainty in turn propagates into the solution of the MKM, including coverages and reaction rates. One of our goals with the UQ is specifically to evaluate the overall impact of errors in each parameter type on the distribution of model responses.

Error distributions for the LSRs have previously been estimated[17] and are used without further adjustment in this work. BEP error distributions for the thermal decomposition reactions were also estimated in prior work[34], and error distributions for the remaining homologous series are estimated in the SI. In performing UQ calculations, the estimated parameters are perturbed within their uncertainty range, and the model is solved. After a sufficient number of replicates (many thousands at least), it is possible to construct a distribution for each of the model responses (rates, selectivities, etc.). We characterize these distributions with representative percentiles: the median and $\pm 34 \%$ bounds (for a normal distribution these correspond to the mean bracketed by one standard deviation).

We also carry out GSA calculations using a variance-based method.[42, 43] We sample points using the radial (or iterated one-factor-at-a-time) method[44, 45], which involves drawing two independent perturbation samples (called the $\mathbf{A}$ and $\mathbf{B}$ vectors). The algorithm then involves solving the model at each of the two independent samples as well as at perturbation samples constructed by replacing one perturbed value at a time in the first set with the corresponding value from the second set (termed the $\mathbf{A}_{\mathbf{B}}$ vector). The fraction of the total model variance attributable to a given parameter can then be estimated using an ANOVA decomposition on the model output (additional details are given in the SI; GSA methods for kinetic modeling have been thoroughly reviewed by Saltelli et al.[46]). We outline the mathematical foundations in the SI.

To efficiently obtain well-converged sensitivity indices at a reduced cost, we employ a two-step procedure. First, we conduct a screening calculation wherein we perturb all parameters but employ fewer total repetitions. From this screening run, parameters with a total sensitivity index larger than 0.02 (i.e., this parameter in combination with all others contributes to at least $2 \%$ of the total variance) are selected for further convergence; all others are subsequently fixed at their nominal values. The second step is to repeat the GSA calculation for the influential parameters using a larger number of repetitions. We have compared the second set to the first and verified qualitative agreement (details in SI).

\section{Activity, Selectivity, and Coverage Maps for the Nominal Model}

Before investigating the effect of uncertainty on model responses, we first demonstrate the predictions made by high throughput MKM. In particular, we construct activity, selectivity, and coverage maps to identify trends in model responses as a function of carbon and oxygen atomic binding energies. Reactor conditions were chosen to ensure relatively low, but not necessarily differential conversions. 

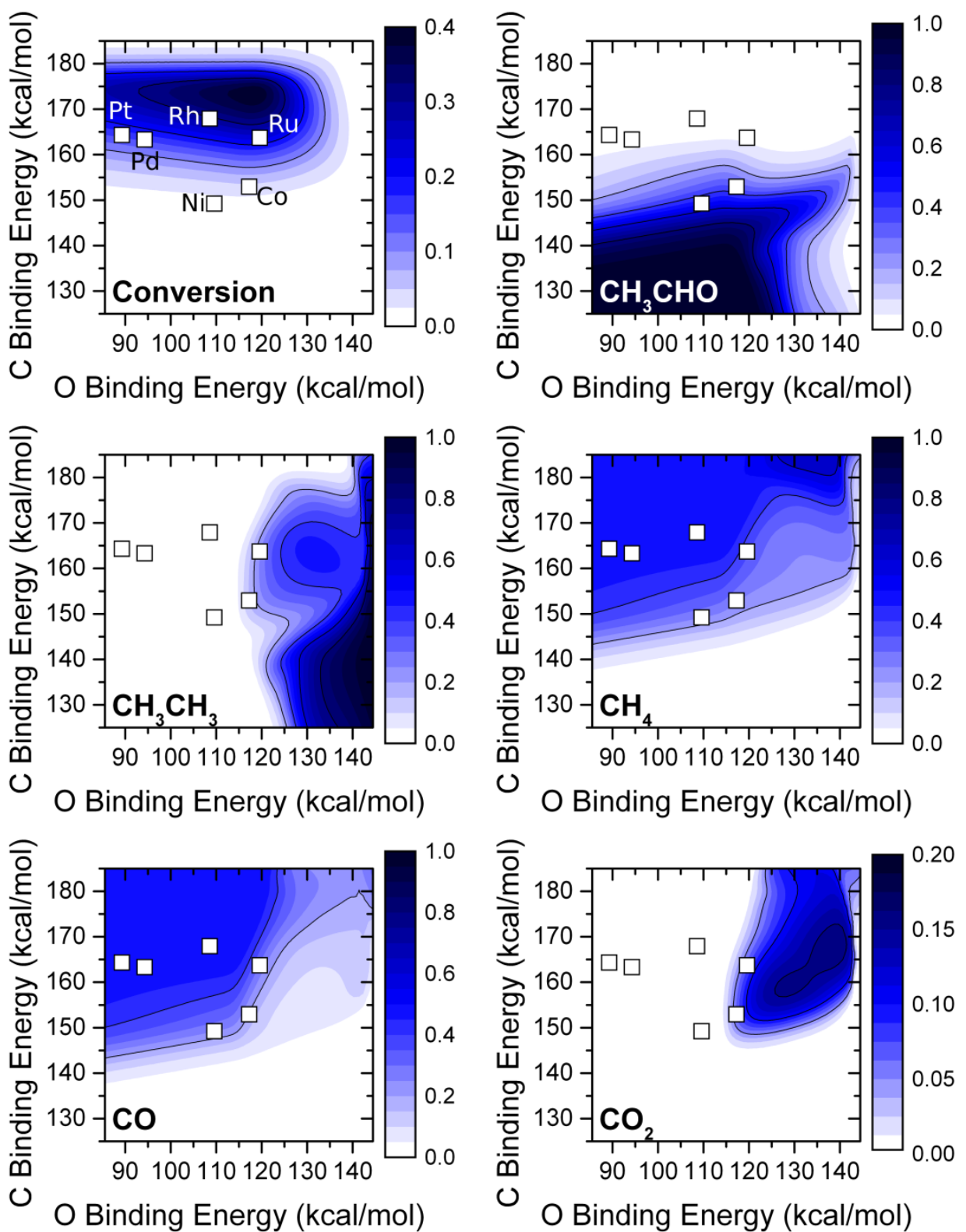

Figure 1. Nominal activity and carbon selectivity maps for ethanol HDO as a function of carbon (C) and oxygen (O) binding energy. Reaction conditions are $573 \mathrm{~K}, 1 \mathrm{~atm}, 12.5 \mathrm{~mol} \%$ ethanol, $37.5 \mathrm{~mol} \% \mathrm{H}_{2}$, bal. $\mathrm{He}$ with $\mathrm{S} / \mathrm{V}=10 \mathrm{~cm}^{-1}$, flow.rate of $1.77 \mathrm{standard} \mathrm{cm}^{3} / \mathrm{min}$, and volume of $1 \mathrm{~cm}^{3}$. Values are fractional. Atomic binding energies for the six closepacked metals are superimposed as white squares on the maps.

Figure 1 shows that activity is maximized at moderate to high $\mathrm{C}$ binding energies and moderate $\mathrm{O}$ binding energies, with the $\mathrm{C}$ binding energy being more important. Four of the single metals lie in the region of moderate to high activity, with $\mathrm{Rh}$ and $\mathrm{Ru}$ being closest to the maximum conversion. With the exception of $\mathrm{O}$ binding energies higher than $140 \mathrm{kcal} / \mathrm{mol}$, there are enough vacant sites present (at least 20\%; Figure 2), and the binding energy, rather than site availability controls the activity. At the high $\mathrm{O}$ binding energies (very oxophilic catalysts), the rapid build-up of $\mathrm{O}$ blocks sites, and site availability becomes a limiting factor. Activity is low in the acetaldehyde region because ethanol binds too weakly and desorbs fast, but as the $\mathrm{C}$ binding energy increases, so does the activity. The impact of the $\mathrm{C}$ binding energy relative to the $\mathrm{O}$ binding energy is consistent with previous findings that the mean binding energy of ethanol-derived species with respect to gas-phase ethanol generally increases with $\mathrm{C}$ binding energy, 
whereas the $\mathrm{O}$ binding energy has a secondary effect.[34] Dehydrogenation to acetaldehyde is preferred at low $\mathrm{O}$ and $\mathrm{C}$ binding energies. Ethane selectivity is maximized at low $\mathrm{C}$ and high $\mathrm{O}$ binding energies, but it is also prevalent at moderate $\mathrm{C}$ and high $\mathrm{O}$ binding energies. As the $\mathrm{C}$ binding energy increases, the product distribution switches from acetaldehyde and ethane to methane and $\mathrm{CO}$ (low $\mathrm{O}$ binding energies) and to a mixture of ethane, methane, and $\mathrm{CO}_{2}$ (high $\mathrm{O}$ binding energies). At high $\mathrm{O}$ and $\mathrm{C}$ binding energies, both $\mathrm{C}-\mathrm{C}$ and $\mathrm{C}-\mathrm{O}$ cracking are active; most of the $\mathrm{CO}$ is directly oxidized to $\mathrm{CO}_{2}$ with $\mathrm{O}$ * produced from the $\mathrm{C}-\mathrm{O}$ cracking. At very high $\mathrm{C}$ binding energies, methane is the dominant hydrocarbon. In the region of high activity, catalytic cracking of ethanol, $\mathrm{CH}_{3} \mathrm{CH}_{2} \mathrm{OH} \rightarrow \mathrm{CH}_{4}+\mathrm{CO}+\mathrm{H}_{2}$, dominates. Ethane, an HDO product, can be produced with high selectivity but at very low conversion. The HDO region is limited to fairly oxophilic materials, in qualitative agreement with prior ethylene glycol predictions.[11] Under no set of atomic binding energies do we see appreciable $\mathrm{C}$ buildup as the concentration of $\mathrm{H}_{2}$ in the feed is sufficient to ensure complete removal of $\mathrm{C}$-containing fragments.

The selectivity trends in Figure 1 are driven primarily by adsorbate thermochemistry (i.e., atomic and species binding energies), rather than kinetics. $\mathrm{C}-\mathrm{C}$ cracking is at the top left (low $\mathrm{O}$ and high $\mathrm{C}$ binding energies). Consistent with the LSR principle, small species binding through $\mathrm{C}$ (e.g., $\mathrm{CO}^{*}$ and $\mathrm{CH}_{\mathrm{x}}{ }^{*}$, see Figure 2 for surface coverages) are preferred in this region where $\mathrm{C}$ binding energies are high. Similarly, dehydrogenation is at the bottom left (low $\mathrm{C}$ and $\mathrm{O}$ binding energies) where acetaldehyde binds too weakly to undergo further reaction once formed. The principal surface species in this region is $\mathrm{H}^{*}$, formed both from the dehydrogenation of ethanol and the presence of $\mathrm{H}_{2}$ in the feed. Finally, C-O cracking to ethane and water is on the lower right (high $\mathrm{O}$ binding energies) where O-containing species are more stable than $\mathrm{C}$-containing species (the $\mathrm{C}_{2}$ hydrocarbons formed rapidly hydrogenate to ethane and desorb). As the $\mathrm{C}$ binding energy increases, $\mathrm{C}-\mathrm{C}$ cracking also becomes important, and $\mathrm{O} *$ is removed via $\mathrm{CO}^{*}$ oxidation (note the high coverages of both $\mathrm{CO}^{*}$ and $\mathrm{O}^{*}$ ). At the extreme top right, when the $\mathrm{C}$ binding energy is high enough to bind the $\mathrm{C}_{2}$ fragments, further decomposition via C-C cracking is possible, and methane, rather than ethane is formed (here the $\mathrm{H}_{2}$ co-feed is vital in the methane formation process). Importantly, single metals all lie in the region where $\mathrm{C}-\mathrm{C}$ cracking is preferred, but $\mathrm{Ru}$ and Co exhibit some deoxygenation activity (C-O cracking). $\mathrm{Ru}$ in particular is an interesting material as it is known to be a good HDO catalyst, e.g.[47]. 

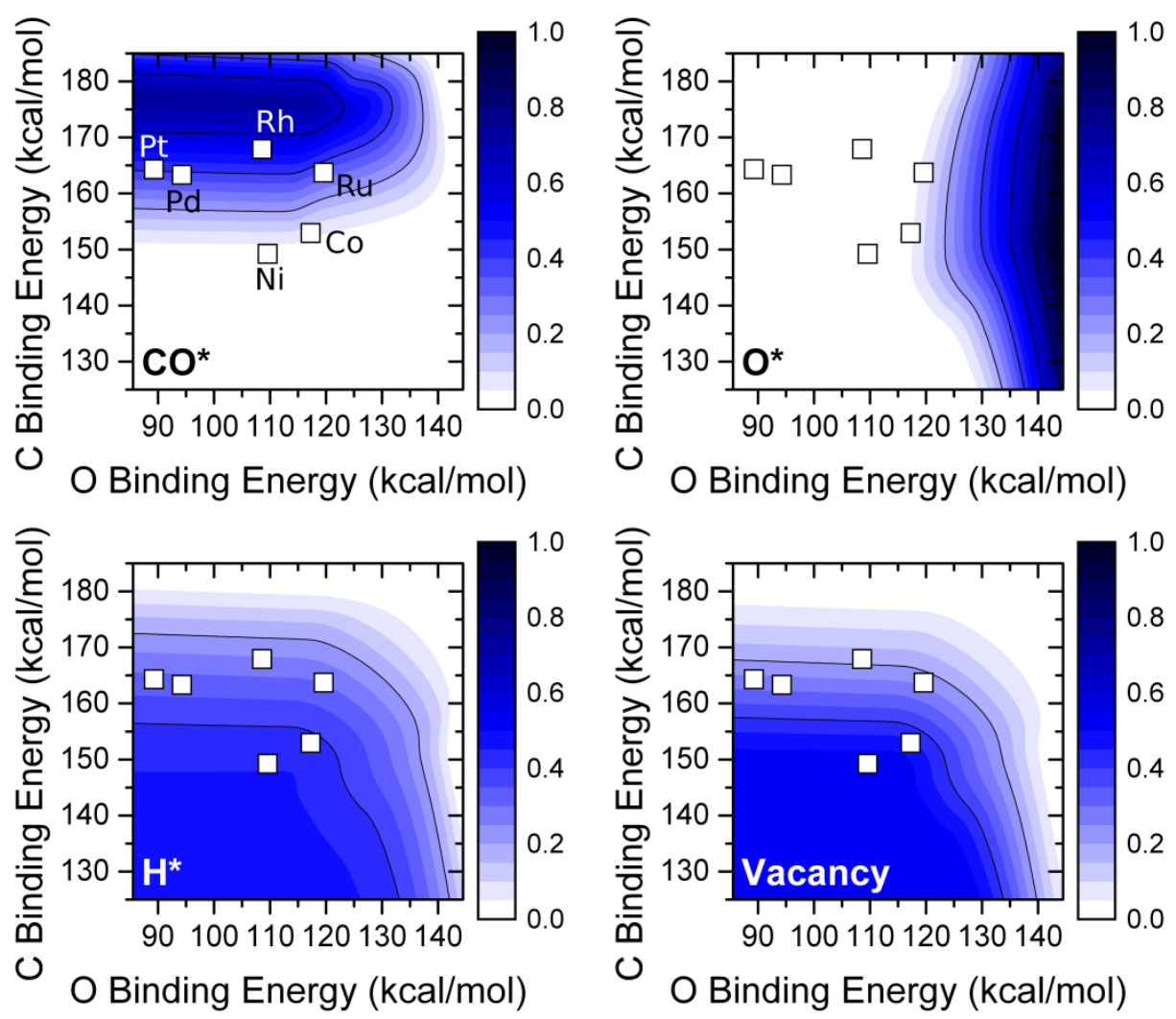

Figure 2. Coverages for dominant species of nominal model. Reaction conditions are the same as in Figure 1 . Very high $\mathrm{C}$ binding energies $(\sim 185 \mathrm{kcal} / \mathrm{mol})$ also have significant quantities of $\mathrm{CCO}^{*}$ (low to moderate $\mathrm{O}$ binding energies) and $\mathrm{CH}^{*}$ (high $\mathrm{O}$ binding energies).

\section{Contributions of LSR and BEP Errors to Uncertainty}

A key question is the reliability of the model predictions. In particular, we wish to investigate the impact of the errors from the LSRs and BEPs on the locations of the maximum activity and selectivity and on the uncertainties in the rates and selectivities. The first is important for guiding experimental tests of proposed catalyst formulations as it gives an estimated range for the atomic binding energies that might give the best performance. The second is vital for understanding whether model rates and selectivities are consistent with experimental data. 

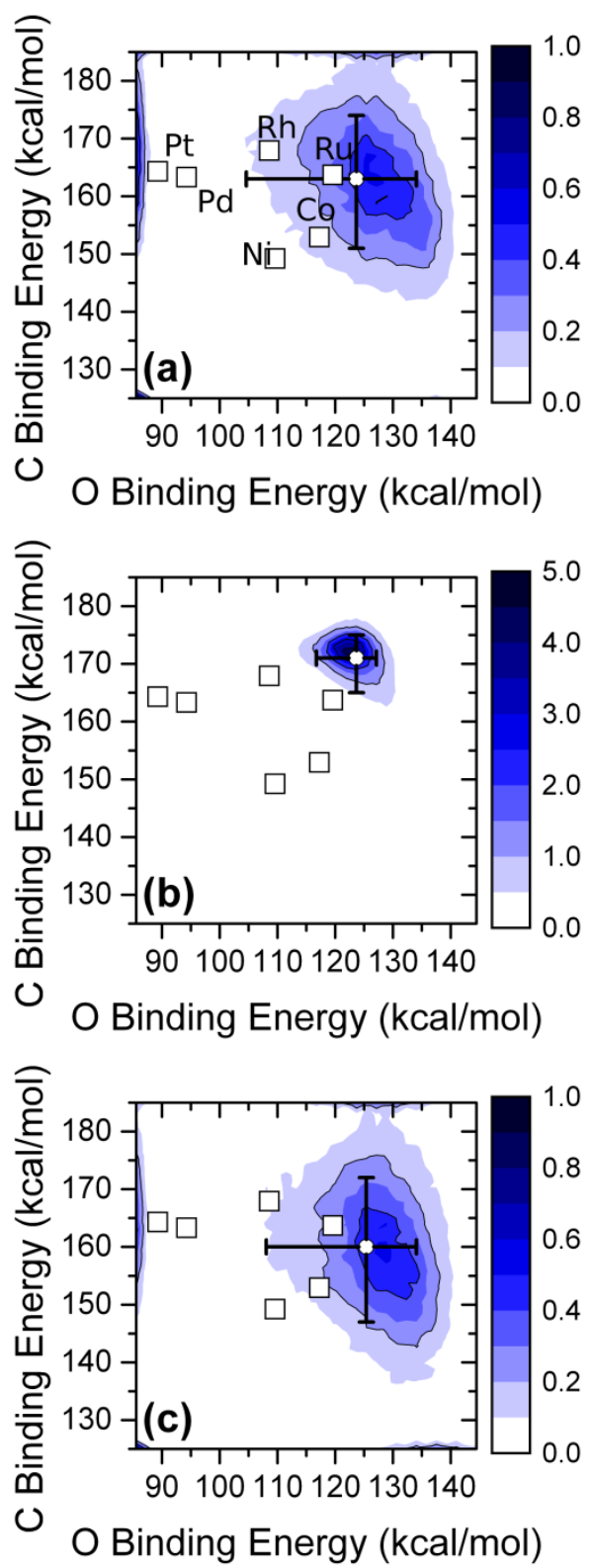

Figure 3. Relative (percent) frequencies with which individual binding energies are the location of the maximum conversion for uncertainties in (a) the linear scaling relations, (b) the Brønsted-Evans-Polanyi relations, and (c) the combination of the two. Reaction conditions are the same as in Figure 1. The median $\mathrm{C}$ and $\mathrm{O}$ binding energies where the conversion is maximized are shown as small open white circles. Error bars on the white circles represent $\pm 34 \%$ confidence intervals in each direction.

We begin by investigating the distribution of the location of maximum conversion, following the procedure of Ulissi et al.[4] Figure 3 shows that uncertainty in the LSRs dominates the distribution of the location of maximum conversion. The distribution of the location of maximum conversion is broad when only the LSR uncertainty is included (Figure 3a). When only BEP uncertainty is employed, the location of maximum conversion is highly localized (Figure $3 \mathrm{~b}$ ) and close to the maximum conversion in the nominal model (Figure 1). When both are combined (Figure 3c), the distribution is very similar to the LSR-only distribution but also shifted somewhat (compare the medians of the binding energies with the maximum conversion indicated by the black squares), indicating that there is a slight nonlinear interaction between the two uncertainties. 
The most likely location for the maximum conversion is significantly shifted from the nominal maximum when ELSR uncertainties are present due to systematic errors in binding energies estimated using the ELSRs.[17]The finding that ELSRs contribute more to the model uncertainty than does the BEP uncertainty was unexpected, owing to the relative sizes of the ELSR and BEP errors. BEP errors can be larger than $1 \mathrm{eV}$, whereas ELSR errors are around an order of magnitude smaller. Furthermore, the BEP error dominates the combined ELSR and BEP uncertainty when the two correlations are used together.[17] We believe that the large contribution of the ELSRs to the model uncertainty is due primarily to the greater importance of adsorbate thermochemistry relative to the kinetics, even in the unperturbed model. The barriers are low enough at the current conditions that other species-related effects are more important. For example, in our previous model of ethanol steam reforming, the rate determining step was found to be the initial dehydrogenation of ethanol because the concentration of adsorbed ethanol was very low.[39] Other compensating effects, such as a large number of parallel reactions or adsorbate interactions (our adsorbate interactions are a function of the atomic binding energies), could also contribute to rendering activation barriers low enough that they do not inhibit overall model rates to the same degree that species binding energies do.

In the case of the combined error distribution, the uncertainty in the location of the binding energy with the highest activity is around $10 \mathrm{kcal} / \mathrm{mol}$, or about $5-10 \%$ of the value of the binding energy (note that the uncertainty is asymmetric, due to the underlying asymmetry of the distribution). The robustness in predicting materials properties for maximum activity is consistent with our earlier work[4] on ammonia decomposition; there, the work focused primarily on uncertainty in pre-exponentials due to using bondorder-conservation for parametrization, which, unlike this work, lacks well-quantifiable errors in energetics.

The locations of maximum selectivity to various major products are extremely robust. Maximum selectivities are achieved at the corners of the map (see Table 3 for median binding energies where selectivity is maximized). For instance, dehydrogenation is maximized at low $\mathrm{C}$ and $\mathrm{O}$ binding energies, $\mathrm{C}-\mathrm{C}$ cracking is maximized at high $\mathrm{C}$ binding energies, and $\mathrm{C}-\mathrm{O}$ cracking is maximized at high $\mathrm{O}$ binding energies. To the best of our knowledge, this is the first time that the effect of errors of fast-screening parameter-estimation methods on selectivity is addressed. Overall, the errors in the correlations have little impact on which materials are predicted to have the highest selectivity, which is an important outcome of this work.

Table 3. Median carbon (C) and oxygen (O) binding energies ( $\mathrm{kcal} / \mathrm{mol})$ of the location of maximum selectivity to major products for each of the sources of error.

\begin{tabular}{cllllll} 
& LSR & \multicolumn{3}{c}{ BEP } & \multicolumn{3}{c}{ LSR+BEP } \\
Species & $\mathrm{C}$ & $\mathrm{O}$ & $\mathrm{C}$ & $\mathrm{O}$ & $\mathrm{C}$ & $\mathrm{O}$ \\
\hline $\mathrm{CH}_{3} \mathrm{CHO}$ & 125.00 & 85.60 & 125.00 & 85.60 & 125.00 & 89.02 \\
$\mathrm{CH}_{3} \mathrm{CH}_{3}$ & 139.00 & 144.45 & 133.00 & 144.45 & 135.00 & 144.45 \\
$\mathrm{CH}_{4}$ & 183.00 & 127.12 & 179.00 & 128.86 & 178.00 & 123.66 \\
$\mathrm{CO}$ & 185.00 & 85.60 & 185.00 & 85.60 & 184.00 & 85.56 \\
$\mathrm{CO}_{2}$ & 174.00 & 134.05 & 168.00 & 130.59 & 171.00 & 134.05
\end{tabular}




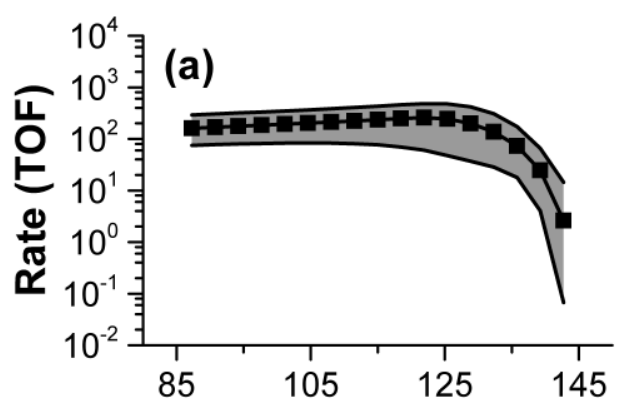

Oxygen Binding Energy (kcal/mol)

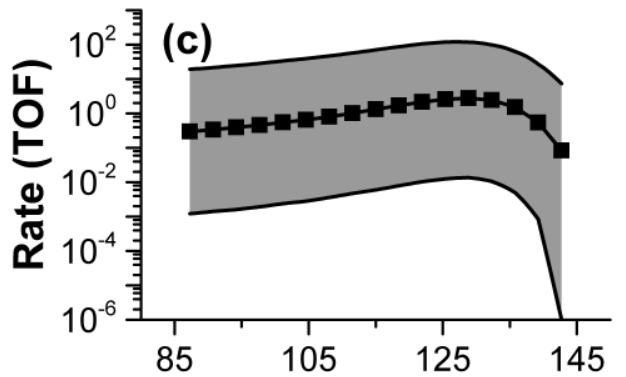

Oxygen Binding Energy (kcal/mol)

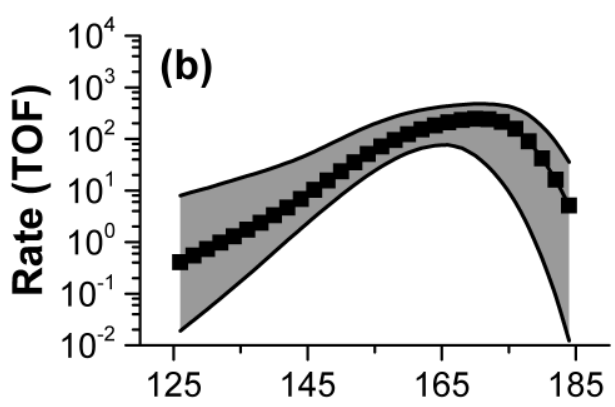

Carbon Binding Energy (kcal/mol)

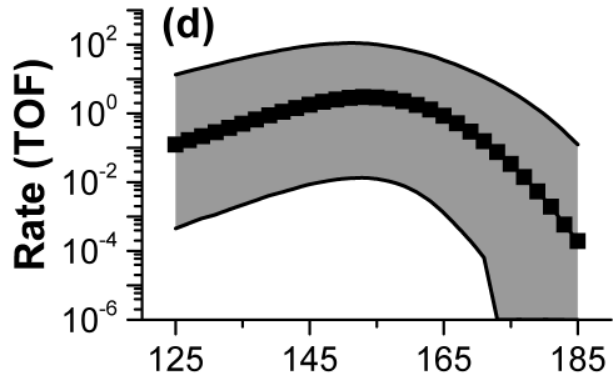

Carbon Binding Energy (kcal/mol)

Figure 4. Uncertainty distributions for reaction rate (turnover frequency in $\mathrm{s}^{-1}$ ) as a function of oxygen binding energy at a carbon binding energy of $150 \mathrm{kcal} / \mathrm{mol}(\mathrm{a}, \mathrm{c})$ and as a function of carbon binding energy at an oxygen binding energy of $130 \mathrm{kcal} / \mathrm{mol}(\mathrm{b}, \mathrm{d})$. Panels (a) and (b) are for BEP uncertainty, and panels (c) and (d) are for combined LSR and BEP uncertainty (LSR-only uncertainty plots are given in the SI due to their similarity to the combined distribution plots). Black lines with symbols are the median $\left(50^{\text {th }}\right.$ percentile) responses, and solid black lines delineate the shaded $\pm 34 \%$ envelope (i.e., $68 \%$ of all responses are within these bounds). For a normal distribution, these percentiles are the mean \pm 1 s.d. After post-processing, some rates were calculated to be identically zero. These were set to $10^{-6}$, which is an order of magnitude smaller than all other lower bounds (e.g., panels $\mathrm{c}$ and $\mathrm{d}$ at the high binding energies).

In addition to the location of the maximum conversion, we also explore the uncertainty distributions for activity and selectivity. Figure 4 examines the uncertainty distributions for the rate (black lines and symbols). The BEP-driven uncertainty is typically less than one order of magnitude around the median and larger near the extreme binding energies (Figure 4a and b). The LSR-driven uncertainty is around 2 and occasionally 3 orders of magnitude around the median (Figure S1), and the LSR uncertainty dominates the combined uncertainty (Figure $4 \mathrm{c}$ and $\mathrm{d}$ ). The uncertainty envelope in carbon selectivities for major products (Figure 5) is generally fairly large. 


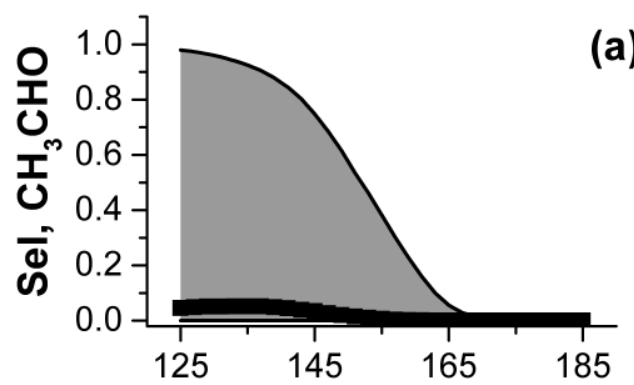

Carbon Binding Energy (kcal/mol)

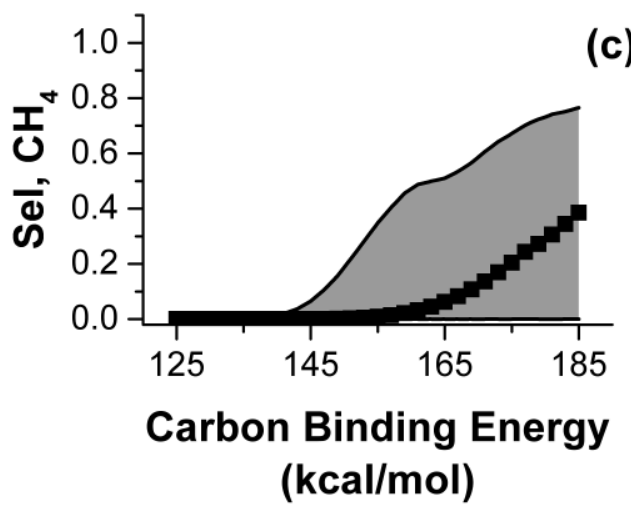

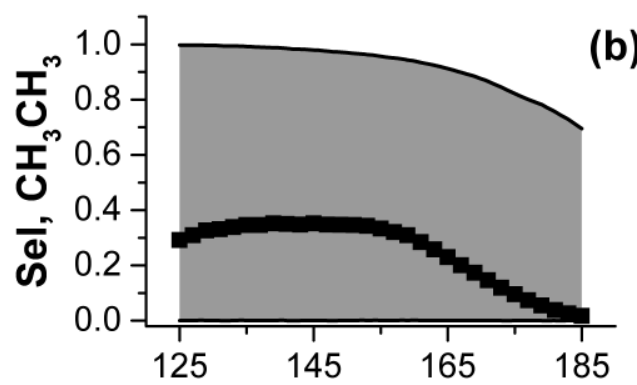

Carbon Binding Energy (kcal/mol)

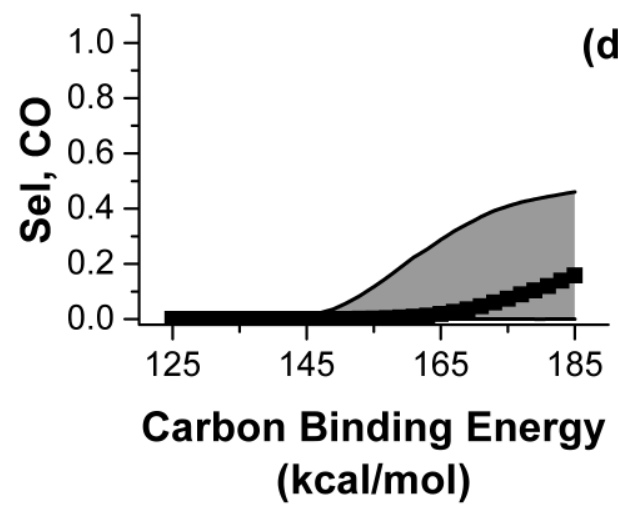

Figure 5. Uncertainty distributions in fractional carbon selectivity for major products as a function of carbon binding energy at an oxygen binding energy of $130 \mathrm{kcal} / \mathrm{mol}$. Black lines with symbols are the median $\left(50^{\text {th }}\right.$ percentile) responses, and solid black lines delineate the shaded $\pm 34 \%$ envelope (i.e., $68 \%$ of all responses are within these bounds). For a normal distribution, these percentiles are the mean \pm 1 s.d.

\section{Analysis of Mechanism and Apparent Kinetics at Representative Binding Energies}

Finally, we apply our UQ and GSA methods to gaining a more detailed mechanistic understanding of the ethanol HDO process. We specifically wish to understand the key differentiating factor between C-C and C-O cracking. To do this, we employ Stochastic Reaction Path Analysis, variance-based GSA, and UQ on the apparent kinetic parameters. 


\section{(a)}

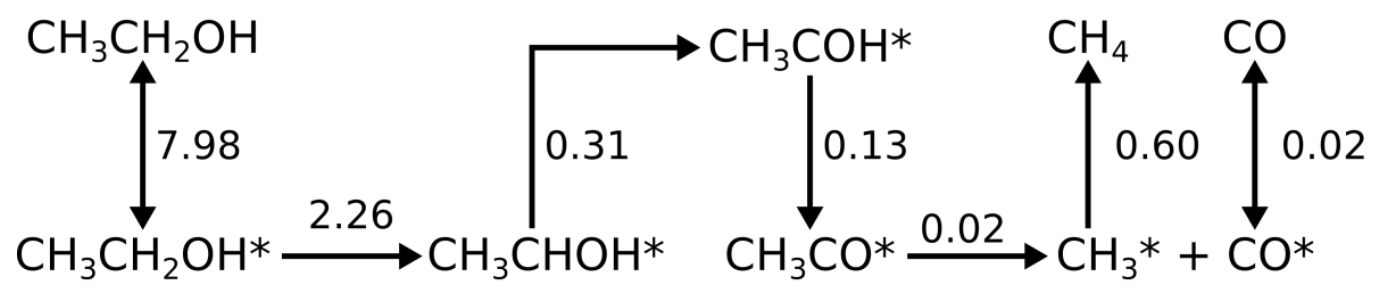

\section{(b)}

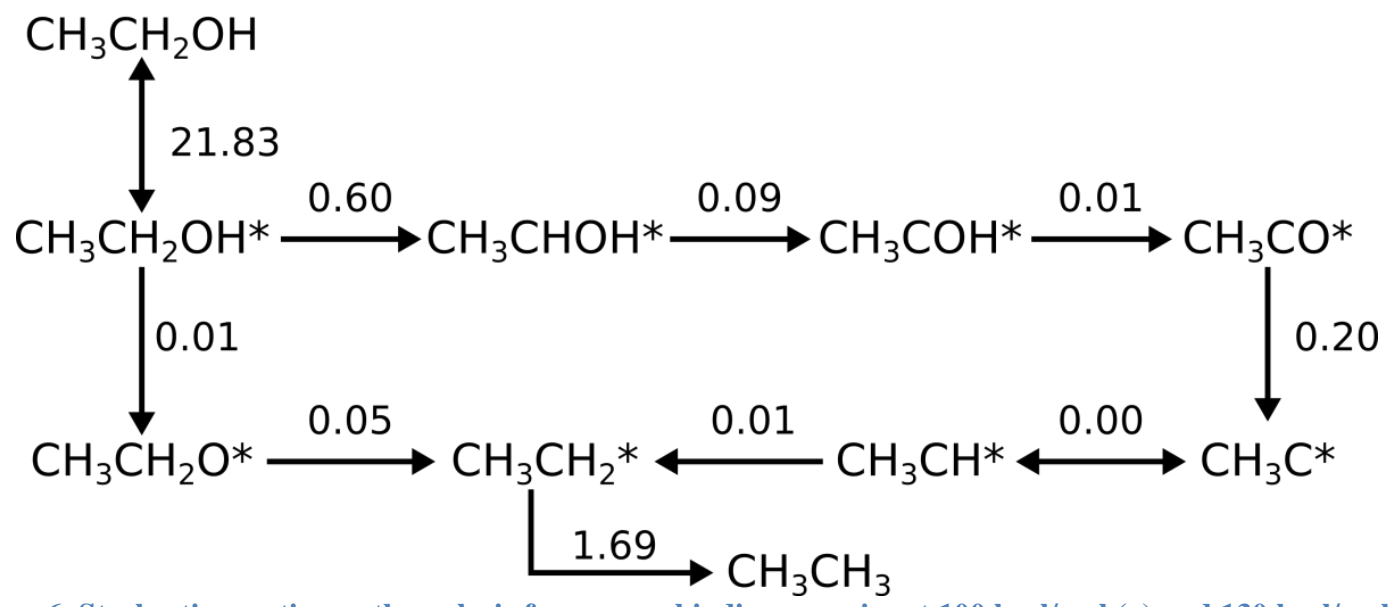

Figure 6. Stochastic reaction path analysis for oxygen binding energies at $100 \mathrm{kcal} / \mathrm{mol}$ (a) and $130 \mathrm{kcal} / \mathrm{mol}$ (b), both at a carbon binding energy of $155 \mathrm{kcal} / \mathrm{mol}$ (i.e., the same binding energies as in Figure 7). Single (double) arrows denote a reversible (irreversible) reaction, as determined by the median partial equilibrium index (PEI). Numbers are median net rates (in $10^{-9} \mathrm{~mol} / \mathrm{s}$ ). Uncertainty ranges for the net rates are provided in the Supporting Information. Because the distributions of the net rates for individual reactions can be quite different, the median net rates leading to and from a given species do not balance as is required in the base model.

In traditional reaction path analysis (RPA), the principal reaction pathways are identified by calculating the amount each reaction contributed to the production or consumption of every species. The stochastic reaction path analysis (SRPA) is a generalization of the traditional RPA to account for the fact we have an ensemble of models (a distribution arising from parametric uncertainty).[27] The goal of a SRPA is to analyze the distribution of species production and consumption rates over the entire ensemble of models. By its nature, the mass balances for each species do not close as they do in a traditional RPA. For the SRPA, we compute medians for the fractional production/consumption (a measure of how much each reaction contributes to the production or consumption of a given species), the rates of each elementary reaction, and the partial equilibrium indices (a measure of the degree of equilibration of an elementary reaction), and then we use the median values to identify the dominant reactions and intermediates. Figure 6 gives the SRPA at the two representative $\mathrm{O}$ binding energies. At the lower $\mathrm{O}$ binding energy (Figure 6a), the mechanism is simple thermal decomposition to $\mathrm{CO}$ and $\mathrm{CH}_{4}$, with $\mathrm{C}-\mathrm{C}$ cracking occurring in $\mathrm{CH}_{3} \mathrm{CO}^{*}$. Our DFT results[34] on several metals suggest C-C scission should actually happen in $\mathrm{CHCO}^{*}$. The difference is probably due to errors in either the thermodynamics (via the LSRs) or the kinetics (via the BEPs). At the higher $\mathrm{O}$ binding energy (Figure 6b), C-O scission occurs either in $\mathrm{CH}_{3} \mathrm{CO}^{*}$ (the principal route) or $\mathrm{CH}_{3} \mathrm{CH}_{2} \mathrm{O}^{*}$ (the secondary route). $\mathrm{H}^{*}$ on the surface then hydrogenates the $\mathrm{C}_{2}$ fragments to ethane, and $\mathrm{O}^{*}$ is removed as water (not shown). 


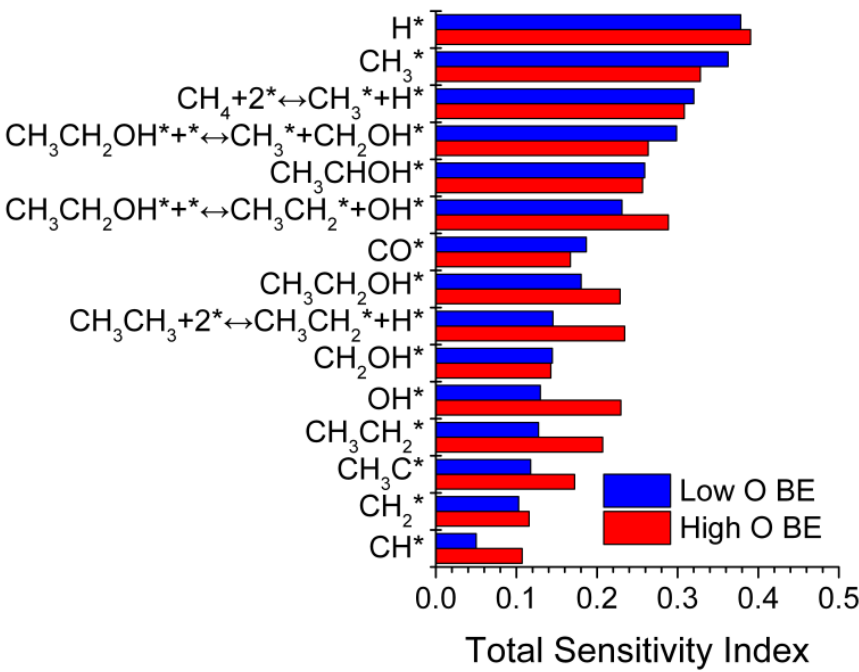

Figure 7. Total sensitivity indices on the conversion at two representative oxygen binding energies of $100 \mathrm{kcal} / \mathrm{mol}$ (low) and $130 \mathrm{kcal} / \mathrm{mol}$ (high) and a C binding energy of $155 \mathrm{kcal} / \mathrm{mol}$. Species and reactions shown are the most influential on conversion. Operating conditions are the same in Figure 1. Species energies are generally more influential than activation energies. Hydrogenations to saturated hydrocarbons, dehydroxylation and C-C bon scission from ethanol are the kinetically key reactions. The high $O$ binding energy mechanism seems to have more parametric interactions (represented by the larger number of parameters with high total sensitivity indices) than the low $\mathbf{O}$ binding energy mechanism.

Figure 7 compares the total sensitivity indices at two representative $\mathrm{O}$ binding energies. The total sensitivity indices (see SI for mathematical definition) measure the fractional contribution of a parameter on its own and in conjunction with all other parameters to the model variance. In the absence of parametric interactions, the sum of all total sensitivity indices is unity. When the sum is larger than unity, as is the case here, parametric interactions are significant. The most important species enthalpies and reaction activation energies for both $\mathrm{O}$ binding energies are identical. The most influential parameters are related to methane formation (via $\mathrm{CH}_{\mathrm{x}}$ enthalpies and methane desorption) and $\mathrm{CO}^{*}$ desorption (controlled by the enthalpy of $\mathrm{CO}^{*}$ ), consistent with the need to remove $\mathrm{C}_{1}$ species from the surface to keep sites open for further reaction. At high $\mathrm{O}$ binding energies, the hydrogenation of $\mathrm{C}_{2}$ species and $\mathrm{OH}^{*}$ play a similar role. The remaining parameters all involve ethanol and its immediate products $\left(\mathrm{CH}_{3} \mathrm{CHOH}^{*}\right.$ and the $\mathrm{C}-\mathrm{C}$ and $\mathrm{C}-\mathrm{O}$ cracking products). Although GSA calculations routinely activate new mechanisms during the global parameter search, the activation energies of the $\mathrm{C}-\mathrm{C}$ and $\mathrm{C}-\mathrm{O}$ cracking reactions in ethanol are surprisingly influential, even though they do not occur either in the SRPA or the nominal model. We believe the reason for this is twofold. First, while the DFT activation energies for these reactions are high enough (more than $1.5 \mathrm{eV}$ ) that they would not ordinarily be expected to be active, the BEPs significantly underpredict them (sometimes by as much as $1 \mathrm{eV}$ ). Second, with the consistently underpredicted activation energy, there is a high probability to achieve an even smaller activation energy via random perturbations, and a new reaction channel opens up, enhancing the total rate. Overall, the similarity of the influential parameters underscores the common mechanism (namely thermal decomposition) present at both binding energies. Where the sensitive parameters differ is after acetyl $\left(\mathrm{CH}_{3} \mathrm{CO} *\right)$. At the lower $\mathrm{O}$ binding energy, the key parameters are related to $\mathrm{C}-\mathrm{C}$ cracking and removal of the resulting products (i.e., $\mathrm{C}_{1}$ species), whereas at the higher $\mathrm{O}$ binding energy, they are related to $\mathrm{C}-\mathrm{O}$ cracking and its resulting products $\left(\mathrm{C}_{2}\right.$ species and water).

Importantly, a larger number of species enthalpies than reaction barriers are important ( $\sim 50$ perturbed species and $\sim 300$ perturbed activation energies, resulting in 11 important species and 4 important reactions), indicating that ethanol decomposition is largely a thermochemistry-controlled process. It also points to the importance of computing and assessing experimentally accurate thermochemistry of select 
species. Importantly, the model can guide experiments, and databases of such information can be developed and shared among mechanisms. For example, the thermochemistry of $\mathrm{H}^{*}, \mathrm{OH}^{*}, \mathrm{CO}^{*}, \mathrm{H}_{2} \mathrm{O}^{*}$ is important (Figure 7); these species are common to numerous reaction mechanisms. Similarly, the $\mathrm{CH}_{\mathrm{x}}{ }^{*}$ $(\mathrm{x}=1-3)$ species are also encountered in several hydrocarbon chemistries.

The relatively large number of parameters with moderate or high total sensitivity indices indicates that parametric interactions are very important. This is underscored by the very small values of the first order sensitivity indices (the maximum value is around 0.03, see SI). Physically, this makes sense. The activity will increase the most when related parameters are varied together. For instance, the enthalpy of $\mathrm{H}^{*}$ is very influential because $\mathrm{H}^{*}$ participates in the majority of the mechanism. Furthermore, the enthalpy of $\mathrm{CH}_{3} *$ and the activation energy of $\mathrm{CH}_{3} *$ hydrogenation are also important. These three parameters $\left(\mathrm{H}^{*}\right.$ and $\mathrm{CH}_{3} *$ enthalpy and $\mathrm{CH}_{3} *$ hydrogenation activation energy) are closely connected. Increasing the enthalpy of $\mathrm{H}^{*}$ and $\mathrm{CH}_{3}{ }^{*}$ together will shift the reaction towards methane, as will lowering the activation energy of the reaction. And obviously, if these changes are made together, the rate of methane formation will increase by more than altering each of them individually, owing to the nonlinear form of the reaction rate expression. The same observation holds for each of the sensitive activation energies; one also finds that if an activation energy is sensitive, then the enthalpies of the associated adsorbates are also sensitive (note that we assumed the gas-phase species enthalpies were exact, and we did not perturb them).

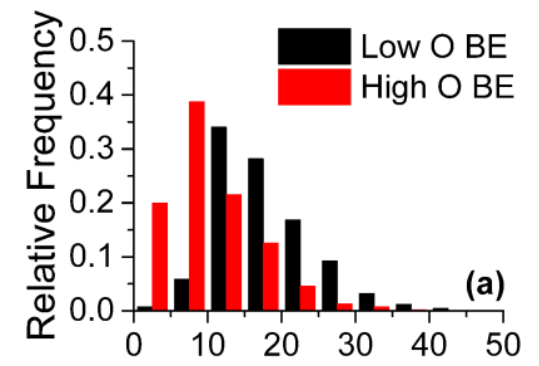

Activation Energy $(\mathrm{kcal} / \mathrm{mol})$

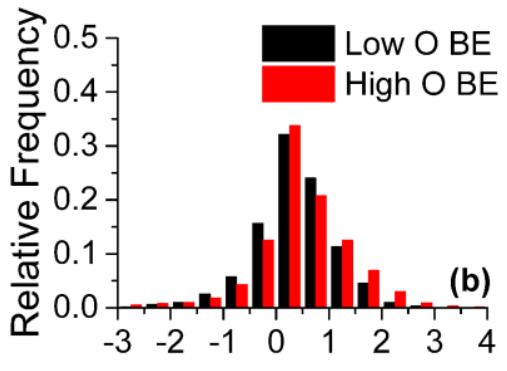

EtOH Reaction Order

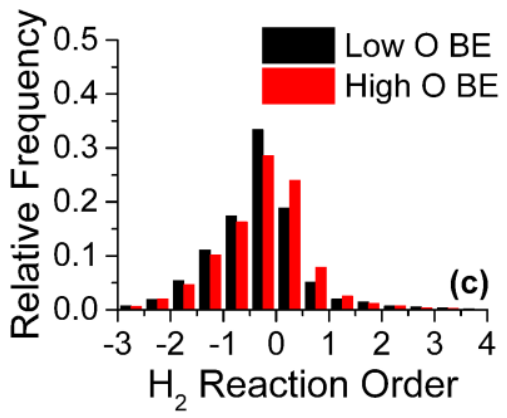

Figure 8. Uncertainty quantification histograms of apparent kinetics: activation energy (a), ethanol reaction order (b), and $\mathrm{H}_{2}$ reaction order (c). Nominal conditions are the same as in Figure 1. Temperatures are in the range $250-400{ }^{\circ} \mathrm{C}$, ethanol concentrations are in the range $1.25 \mathrm{~mol} \%$ - $25 \mathrm{~mol} \%$, and $\mathrm{H}_{2}$ concentrations are in the range $3.75 \mathrm{~mol} \%$ - 75 $\mathrm{mol} \%$. C binding energy is $155 \mathrm{kcal} / \mathrm{mol}$. O binding energies (BE) are $100 \mathrm{kcal} / \mathrm{mol}$ and $130 \mathrm{kcal} / \mathrm{mol}$ (labeled as low and high, respectively).

We are also interested in the distributions of the apparent kinetic parameters, specifically the apparent activation energy and the apparent ethanol and hydrogen reaction orders. We generate distributions of the apparent kinetic parameters by quantifying the uncertainty in the rate at a series of feed conditions (temperature and composition) with the same set of sampled parameter perturbations for each feed condition. Apparent kinetic parameters are then fit for each set of rates (one set per perturbation). From the raw set of sampled apparent kinetic parameters, a distribution can be constructed. We represent these as histograms of relative frequencies as a function of the activation energy or reaction order. Relative frequencies are calculated by counting the number of times an activation energy or reaction order falls within a specified parameter range (or bin) and normalizing by the total number of sample points. The most likely activation energies (i.e., those with the highest relative frequency) are around $10 \mathrm{kcal} / \mathrm{mol}$, with the higher $\mathrm{O}$ binding energy model having a slightly lower activation energy, consistent with its inherently higher activity. In both cases, the upper bound on the activation energy is around $20 \mathrm{kcal} / \mathrm{mol}$. The higher activity at the higher $\mathrm{O}$ binding energy arises from the stronger binding of the terminal $\mathrm{O}$ in ethanol (the $\mathrm{C}$ binding energy is the same in both histograms).

The ethanol reaction orders are nearly identical and positive with most values falling between zero and one, indicating that both mechanisms share a common initial path of thermal decomposition. In both cases, there are also a large fraction of states with negative reaction orders. These negative reaction orders 
most likely are the result of combined strongly-bound species blocking free sites, which are required for decomposition to occur, with high decomposition barriers, making removal of such species slow. The total number of parameter sets with negative orders depends strongly on the $\mathrm{O}$ binding energy. At the lower $\mathrm{O}$ binding energy, the $\mathrm{H}_{2}$ order is strongly negative (most values are less than zero, with only a few slightly larger than zero). Consistent with the already high $\mathrm{H}^{*}$ coverage (see Figure 1), additional $\mathrm{H}^{*}$ blocks sites as well as reduces the driving force to dehydrogenation of ethanol. At the higher O binding energy, additional $\mathrm{H}_{2}$ has a slightly more positive order. This is consistent with the additional $\mathrm{H}^{*}$ acting both to inhibit ethanol dehydrogenation as well as removing $\mathrm{O}$ (as water), which tends to produce free sites necessary for the conversion of ethanol. These results also indicate that a change in apparent reaction orders among materials may occur. In comparing the apparent reaction orders to experimental data, one should keep in mind that idealized materials may behave differently from real materials. For example, the catalyst support or promoter may be effective at providing an alternate path for removing products from the active phase of the catalyst, resulting in free active sites and the reduction or elimination of site blocking that leads to negative reaction orders. Qualitative differences from experimental data underscores potential limitations and areas for improvement of the model.

\section{Conclusions}

We introduced the first-of-its kind uncertainty quantification (UQ) and global sensitivity analysis (GSA) to gain insights into the impact of errors of linear scaling relations (LSRs) and Bronsted-EvansPolanyi (BEP) correlatons on the predictive ability of high throughput microkinetic models. We have applied the methodology to ethanol hydrodeoxygenation (HDO). We show that the activity and selectivity depend strongly on $\mathrm{C}$ and $\mathrm{O}$ binding energies, in qualitatiave agreement with previous models on $\mathrm{HDO}$ of oxygenates. We find that common single metals lie in the region of simple thermal decomposition to $\mathrm{C}_{1}$ products $\left(\mathrm{CO}\right.$ and $\left.\mathrm{CH}_{4}\right)$, with the exception of $\mathrm{Ru}$, which is near the transition between $\mathrm{C}_{1}$ and $\mathrm{C}_{2}$ products (e.g., $\mathrm{C}_{2} \mathrm{H}_{6}$ ). More oxophilic materials, e.g., $\mathrm{WC}$ and $\mathrm{Mo}_{2} \mathrm{C}$, are needed for $\mathrm{HDO}$ to hydrocarbons; yet, the activity of such materials will be inherently low compared to the best materials for thermal decomposition. Interestingly, the preference towards $C_{1}$ vs. $C_{2}$ products is driven primarily by thermochemistry of adsorbates rather than kinetics. By applying UQ and GSA methods, we were also able to show that ethanol HDO has a common dehydrogenation mechanism leading to the formation of $\mathrm{CH}_{3} \mathrm{CO}^{*}$. The selectivity to $\mathrm{C}_{1}$ vs. $\mathrm{C}_{2}$ products is controlled primarily by the stability of the products of the decomposition of $\mathrm{CH}_{3} \mathrm{CO}^{*}$.

Uncertainties in the LSRs employed for estimating adsorbate enthalpies, rather than BEPs, are the dominant contributor to uncertainty in model predictions. The location of peak conversion can be estimated to within about $10 \mathrm{kcal} / \mathrm{mol}$ with current methods. This uncertainty is less than $10 \%$ of the optimal properties, i.e., the error in predictions is relatively low. Materials properties for selectivities are very robust. In contrast, uncertainties in model rates can differ up to 2-3 orders of magnitude from the median. This high uncertainty is consistent with but higher that that in recent work $[26,27]$ assessing the role of DFT errors on the rate in a DFT-based microkinetic model. As mentioned earlier, even the best available DFT functionals still show systematic errors with respect to experiments. These systematic errors will propagate to materials predictions, and improved DFT methods should be able to minimize this effect. Due to errors in the LSRs, the medians do not always closely track the nominal rate. By comparison, uncertainties in rates are less than one order of magnitude when only BEP error is accounted for.

Overall, despite the uncertainty in parameter estimation using LSRs and BEPs, the models are sufficient to guide materials selection. More accurate LSRs are needed to reduce model uncertainty, especially absolute values of rates and selectivities. In contrast, current BEP methods appear to introduce relatively little uncertainty into model predictions and very little systematic error. As a long term need, we propose to develop more accurate LSRs. As a short term alternative, one could use the existing BEPs and supply DFT-based thermochemistry for certain materials to refine specific points on these maps. Given the much lower cost of computing thermochemistry than barriers this may be a reasonbale compromise. 
An even more appealing approach we propose is to employ hierarchical refinement, i.e., predict maps using LSRs and BEPs and refine the important thermochemistry only (obtained via LSRs) using DFT. This is a very doable task.

\section{Supporting Information}

Supporting Information on DFT calculations (including total energies and images of converged structures), BEP correlations, variance-based global sensitivity analysis, and UQ maps for the location of maximum conversion in the presence of LSR only errors is available.

\section{Acknowledgments}

The ethanol mechanism and the DFT calculations were supported from the Catalysis Center for Energy Innovation (CCEI), an Energy Frontier Research Center funded by the U.S. Department of Energy, Office of Science, Office of Basic Energy Sciences under award number DE-SC0001004. The uncertainty analysis was supported by the U.S.Department of Energy Office of Science, Office of Advanced Scientific Computing Research, and Applied Mathematics program under Award No. DESC0010549.The authors gratefully acknowledge computer time from the Center for Functional Nanomaterials, Brookhaven National Laboratory, supported by the U.S. Department of Energy, Office of Basic Energy Sciences under Contract No. DE-AC02-98CH10886.

\section{References}

[1] D. Farrusseng, High-throughput heterogeneous catalysis, Surface Science Reports, 63 (2008) 487-513.

[2] M. Salciccioli, M. Stamatakis, S. Caratzoulas, D.G. Vlachos, A review of multiscale modeling of metalcatalyzed reactions: Mechanism development for complexity and emergent behavior, Chemical Engineering Science, 66 (2011) 4319-4355.

[3] A. Logadottir, T. Rod, J. Norskov, B. Hammer, S. Dahl, C. Jacobsen, The Bronsted-Evans-Polanyi relation and the volcano plot for ammonia synthesis over transition metal catalysts, Journal Of Catalysis, 197 (2001) 229-231.

[4] Z. Ulissi, V. Prasad, D.G. Vlachos, Effect of multiscale model uncertainty on identification of optimal catalyst properties, Journal Of Catalysis, 281 (2011) 339-344.

[5] T. Bligaard, J. Norskov, S. Dahl, J. Matthiesen, C. Christensen, J. Sehested, The Bronsted-EvansPolanyi relation and the volcano curve in heterogeneous catalysis, Journal Of Catalysis, 224 (2004) 206217.

[6] M. Andersson, T. Bligaard, A. Kustov, K. Larsen, J. Greeley, T. Johannessen, C. Christensen, J. Norskov, Toward computational screening in heterogeneous catalysis: Pareto-optimal methanation catalysts, Journal of Catalysis, 239 (2006) 501-506.

[7] J. Cheng, P. Hu, Utilization of the three-dimensional volcano surface to understand the chemistry of multiphase systems in heterogeneous catalysis., J. Am. Chem. Soc., 130 (2008) 10868-10869.

[8] G. Jones, J. Jakobsen, S. Shim, J. Kleis, M. Andersson, J. Rossmeisl, F. Abild-pedersen, T. Bligaard, S. Helveg, B. Hinnemann, J. Rostrup-nielsen, I. Chorkendorff, J. Sehested, J. Norskov, First principles calculations and experimental insight into methane steam reforming over transition metal catalysts, Journal Of Catalysis, 259 (2008) 147-160.

[9] J. Greeley, J. Norskov, Combinatorial Density Functional Theory-Based Screening of Surface Alloys for the Oxygen Reduction Reaction, Journal Of Physical Chemistry C, 113 (2009) 4932-4939.

[10] P. Ferrin, D. Simonetti, S. Kandoi, E. Kunkes, J. Dumesic, J. Norskov, M. Mavrikakis, Modeling Ethanol Decomposition on Transition Metals: A Combined Application of Scaling and Bronsted-EvansPolanyi Relations, Journal Of The American Chemical Society, 131 (2009) 5809-5815. 
[11] M. Salciccioli, D.G. Vlachos, Kinetic Modeling of Pt Catalyzed and Computation-Driven Catalyst Discovery for Ethylene Glycol Decomposition, ACS Catalysis, 1 (2011) 1246-1256.

[12] A.J. Medford, A. Vojvodic, J.S. Hummelshøj, J. Voss, F. Abild-Pedersen, F. Studt, T. Bligaard, A. Nilsson, J.K. Nørskov, From the Sabatier principle to a predictive theory of transition-metal heterogeneous catalysis, Journal Of Catalysis, 328 (2015) 36-42.

[13] F. Abild-Pedersen, J. Greeley, F. Studt, J. Rossmeisl, T.R. Munter, P.G. Moses, E. Skulason, T. Bligaard, J.K. Norskov, Scaling properties of adsorption energies for hydrogen-containing molecules on transition-metal surfaces, Phys. Rev. Lett., 99 (2007) 016105.

[14] E.M. Fernández, P.G. Moses, A. Toftelund, H.A. Hansen, J.I. Martínez, F. Abild-Pedersen, J. Kleis, B. Hinnemann, J. Rossmeisl, T. Bligaard, J.K. Nørskov, Scaling relationships for adsorption energies on transition metal oxide, sulfide, and nitride surfaces., Angew. Chem. Int. Ed. Engl., 47 (2008) 4683-4686.

[15] M. Salciccioli, Y. Chen, D. Vlachos, Density Functional Theory-Derived Group Additivity and Linear Scaling Methods for Prediction of Oxygenate Stability on Metal Catalysts: Adsorption of Open-Ring Alcohol and Polyol Dehydrogenation Intermediates on Pt-Based Metals, Journal Of Physical Chemistry C, 114 (2010) 20155-20166.

[16] G. Jones, F. Studt, F. Abild-Pedersen, J.K. Norskov, T. Bligaard, Scaling relationships for adsorption energies of C-2 hydrocarbons on transition metal surfaces, Chemical Engineering Science, 66 (2011) 6318-6323.

[17] J.E. Sutton, D.G. Vlachos, Error estimates in semi-empirical estimation methods of surface reactions, Journal Of Catalysis, 297 (2013) 202-216.

[18] E. Shustorovich, A. Zeigarnik, The UBI-QEP method: Basic formalism and applications to chemisorption phenomena on transition metal surfaces. Chemisorption energetics, Russian Journal Of Physical Chemistry, 80 (2006) 4-30.

[19] M. Maestri, K. Reuter, Semiempirical Rate Constants for Complex Chemical Kinetics: First-Principles Assessment and Rational Refinement, Angewandte Chemie International Edition, 50 (2011) 1194-1197.

[20] V. Pallassana, M. Neurock, Electronic factors governing ethylene hydrogenation and dehydrogenation activity of pseudomorphic Pd-ML/Re(0001), Pd-ML/Ru(0001), Pd(111), and Pd$\mathrm{ML} / \mathrm{Au}(111)$ surfaces, Journal of Catalysis, 191 (2000) 301-317.

[21] R. Alcala, M. Mavrikakis, J. Dumesic, DFT studies for cleavage of C-C and C-O bonds in surface species derived from ethanol on Pt(111), Journal of Catalysis, 218 (2003) 178-190.

[22] A. Michaelides, Z. Liu, C. Zhang, A. Alavi, D. King, P. Hu, Identification of general linear relationships between activation energies and enthalpy changes for dissociation reactions at surfaces, Journal Of The American Chemical Society, 125 (2003) 3704-3705.

[23] R. Van SANTEN, W. OFFERMANS, K. MALEK, E. PIDKO, Computational modeling of catalytic reactivity, Molecular Simulation, 33 (2007) 327-336.

[24] J.E. Sutton, D.G. Vlachos, A Theoretical and Computational Analysis of Linear Free Energy Relations for the Estimation of Activation Energies, ACS Catalysis, 2 (2012) 1624-1634.

[25] J.E. Sutton, D.G. Vlachos, Building large microkinetic models with first-principles' accuracy at reduced computational cost, Chemical Engineering Science, 121 (2015) 190-199.

[26] A.J. Medford, J. Wellendorff, A. Vojvodic, F. Studt, F. Abild-Pedersen, K.W. Jacobsen, T. Bligaard, J.K. Nørskov, Assessing the reliability of calculated catalytic ammonia synthesis rates, Science, 345 (2014) 197-200.

[27] J.E. Sutton, W. Guo, M.A. Katsoulakis, D.G. Vlachos, Effects of correlated parameters and uncertainty in electronic-structure-based chemical kinetic modelling, Nature Chemistry, (2016).

[28] D. Loffreda, F.c. Delbecq, F. Vigné, P. Sautet, Fast prediction of selectivity in heterogeneous catalysis from extended Brønsted-Evans-Polanyi relations: a theoretical insight., Angew. Chem. Int. Ed. Engl., 48 (2009) 8978-8980. 
[29] J. Zaffran, C. Michel, F. Auneau, F. Delbecq, P. Sautet, Linear Energy Relations As Predictive Tools for Polyalcohol Catalytic Reactivity, Acs Catalysis, 4 (2014) 464-468.

[30] J. Zaffran, C. Michel, F. Delbecq, P. Sautet, Trade-Off between Accuracy and Universality in Linear Energy Relations for Alcohol Dehydrogenation on Transition Metals, The Journal of Physical Chemistry C, 119 (2015) 12988-12998.

[31] S. Wang, V. Petzold, V. Tripkovic, J. Kleis, J.G. Howalt, E. Skúlason, E.M. Fernández, B. Hvolb\aek, G. Jones, A. Toftelund, H. Falsig, M. Björketun, F. Studt, F. Abild-Pedersen, J. Rossmeisl, J.K. Nørskov, T. Bligaard, Universal transition state scaling relations for (de)hydrogenation over transition metals., Phys Chem Chem Phys, 13 (2011) 20760-20765.

[32] A. Mhadeshwar, D. Vlachos, Hierarchical, multiscale surface reaction mechanism development: CO and $\mathrm{H}-2$ oxidation, water-gas shift, and preferential oxidation of $\mathrm{CO}$ on Rh, Journal of Catalysis, 234 (2005) 48-63.

[33] J. Soler, E. Artacho, J. Gale, A. García, J. Junquera, P. Ordejón, D. Sánchez-Portal, The SIESTA method for ab initio order- $N$ materials simulation, J Phys: Condens Matter, 14 (2002) 2745-2779.

[34] J.E. Sutton, D.G. Vlachos, Ethanol Activation on Closed-Packed Surfaces, INDUSTRIAL \& ENGINEERING CHEMISTRY RESEARCH, 54 (2015) 4213-4225.

[35] J.P. Perdew, K. Burke, M. Ernzerhof, Generalized gradient approximation made simple, Physical Review Letters, 77 (1996) 3865-3868.

[36] J. Wellendorff, T.L. Silbaugh, D. Garcia-Pintos, J.K. Nørskov, T. Bligaard, F. Studt, C.T. Campbell, A benchmark database for adsorption bond energies to transition metal surfaces and comparison to selected DFT functionals, Surface Science, 640 (2015) 36-44.

[37] A. Alavi, P. Hu, T. Deutsch, P.L. Silvestrelli, J. Hutter, CO Oxidation on Pt(111): An Ab Initio Density Functional Theory Study, Physical Review Letters, 80 (1998) 3650-3653.

[38] H. Wang, Z. Liu, Comprehensive mechanism and structure-sensitivity of ethanol oxidation on platinum: New transition-state searching method for resolving the complex reaction network, Journal Of The American Chemical Society, 130 (2008) 10996-11004.

[39] J.E. Sutton, P. Panagiotopoulou, X.E. Verykios, D.G. Vlachos, Combined DFT, Microkinetic, and Experimental Study of Ethanol Steam Reforming on Pt, The Journal of Physical Chemistry C, 117 (2013) 4691-4706.

[40] S. Wang, V. Vorotnikov, J.E. Sutton, D.G. Vlachos, Bronsted-Evans-Polanyi and Transition State Scaling Relations of Furan Derivatives on Pd(111) and Their Relation to Those of Small Molecules, Acs Catalysis, 4 (2014) 604-612.

[41] A.B. Mhadeshwar, H. Wang, D.G. Vlachos, Thermodynamic consistency in microkinetic development of surface reaction mechanisms, Journal of Physical Chemistry B, 107 (2003) 12721-12733.

[42] I.M. Sobol, Global sensitivity indices for nonlinear mathematical models and their Monte Carlo estimates, Math. Comput. Simul., 55 (2001) 271-280.

[43] A. Saltelli, Making best use of model evaluations to compute sensitivity indices, Computer Physics Communications, 145 (2002) 280-297.

[44] A. Saltelli, P. Annoni, I. Azzini, F. Campolongo, M. Ratto, S. Tarantola, Variance based sensitivity analysis of model output. Design and estimator for the total sensitivity index, Computer Physics Communications, 181 (2010) 259-270.

[45] F. Campolongo, A. Saltelli, J. Cariboni, From screening to quantitative sensitivity analysis. A unified approach, Computer Physics Communications, 182 (2011) 978-988.

[46] A. Saltelli, M. Ratto, S. Tarantola, F. Campolongo, Update 1 of: Sensitivity Analysis for Chemical Models, CHEMICAL REVIEWS, 112 (2012) PR1-PR21.

[47] J. Jae, W. Zheng, R.F. Lobo, D.G. Vlachos, Production of Dimethylfuran from Hydroxymethylfurfural through Catalytic Transfer Hydrogenation with Ruthenium Supported on Carbon, ChemSusChem, 6 (2013) 1158-1162. 